\title{
Integrable open spin chains from flavored ABJM theory
}

\section{Nan Bai, ${ }^{a}$ Hui-Huang Chen, ${ }^{a, b}$ Song He, ${ }^{c, d}$ Jun-Bao Wu, ${ }^{e, f, g}$ Wen-Li Yang ${ }^{h, i}$ and Meng-Qi Zhu ${ }^{j}$}

${ }^{a}$ Institute of High Energy Physics, and Theoretical Physics Center for Science Facilities, Chinese Academy of Sciences, 19B Yuquan Road, Beijing, 100049 China

${ }^{b}$ University of Chinese Academy of Sciences, $19 A$ Yuquan Road, Beijing, 100049 China

${ }^{c}$ Max Planck Institute for Gravitational Physics (Albert Einstein Institute), Am Mühlenberg 1, Golm, 14476 Germany

${ }^{d}$ CAS Key Laboratory of Theoretical Physics, Institute of Theoretical Physics, Chinese Academy of Sciences, 55 Zhong Guan Cun East Road, Beijing, 100190 China

${ }^{e}$ School of Science, Tianjin University, 92 Weijin Road, Tianjin, 300072 China

${ }^{f}$ School of Physics and Nuclear Energy Engineering, Beihang University, 37 Xueyuan Road, Beijing, 100191 China

${ }^{g}$ Center for High Energy Physics, Peking University, 5 Yiheyuan Road, Beijing, 100871 China

${ }^{h}$ Institute of Modern Physics, Northwest University, 229 Taibai Beilu, Xian, 710069 China

${ }^{i}$ Shaanxi Key Laboratory for Theoretical Physics Frontiers, 229 Taibai Beilu, Xian, 710069 China

${ }^{j}$ International School of Advanced Studies (SISSA) and INFN, Sezione di Trieste, via Bonomea 265, Trieste, 34136 Italy

E-mail: bainan@ihep.ac.cn, chenhh@ihep.ac.cn, hesong17@gmail.com, junbao.wu@tju.edu.cn, wlyang@nwu.edu.cn, mzhu@sissa.it

ABSTRACT: We compute the two-loop anomalous dimension matrix in the scalar sector of planar $\mathcal{N}=3$ flavored ABJM theory. Using coordinate Bethe ansatz, we obtain the reflection matrices and confirm that the boundary Yang-Baxter equations are satisfied. This establishes the integrability of this theory in the scalar sector at the two-loop order.

Keywords: Chern-Simons Theories, Integrable Field Theories

ARXIV EPRINT: 1704.05807 


\section{Contents}

1 Introduction 1

2 The action of $\mathcal{N}=3$ flavored ABJM theory 3

2.1 The action in $\mathcal{N}=2$ superfield formulation 3

2.2 The action in $\mathcal{N}=2$ component field formulation 4

2.3 The action in $\mathcal{N}=3$ component field formulation 5

3 Two-loop perturbative calculations and the Hamiltonian $\quad 7$

$\begin{array}{lll}3.1 & \text { Boundary three-site scalar interactions } & 7\end{array}$

$\begin{array}{llr}3.2 & \text { Boundary two-site Yukawa type interactions } & 9\end{array}$

$\begin{array}{ll}3.3 \text { The two-loop Hamiltonian } & 10\end{array}$

4 Integrability from coordinate Bethe ansatz $\quad 11$

$\begin{array}{lll}4.1 & \text { Two-particle mixed sector } & 13\end{array}$

$\begin{array}{lll}4.2 & \text { Four-particle mixed sector } & 15\end{array}$

5 Conclusion and discussions 18

A Some details of $\mathcal{N}=2$ formulation $\quad 20$

$\begin{array}{lll}\text { A.1 The on-shell values of auxiliary fields } & 20\end{array}$

A.2 The potential terms in $\mathcal{N}=2$ formulation $\quad 21$

B BPS property of the reference state $\quad 22$

$\begin{array}{ll}\text { C The bulk S-matrix } & 23\end{array}$

\section{Introduction}

Integrability in planar four-dimensional $\mathcal{N}=4$ super Yang-Mills theory [1] and threedimensional ABJM theory [2] makes the non-perturbative computations in the planar limit possible even for some non-supersymmetric quantities [3]. In both cases, the single trace gauge invariant composite operators are mapped to states on a closed spin chain and the anomalous dimension matrix (ADM) of these operators can be mapped to Hamiltonian of the spin chain. The first evidence of the integrability came from the fact that the Hamiltonians in the scalar sector obtained from the leading-order perturbation theory are integrable [4-6].

In the four dimensional case, one can add flavors to this $\mathcal{N}=4$ super Yang-Mills theory or first perform some orientifold projections and then add certain flavors to obtain $\mathcal{N}=2$ supersymmetric gauge theories. Here by flavors, we mean matter fields in the 
fundamental or anti-fundamental representation of the gauge group. It was found that both theories with flavors are integrable at one-loop order [7]-[9]. In these cases, the single trace operators built only with fields in the adjoint representation of the gauge group are still mapped to states of a closed spin chain. There are also gauge invariant composite operators with fields in the (anti-)fundamental representation in two ends and fields in the adjoint representation in the bulk. ${ }^{1}$ These operators are mapped to states of an open spin chain.

In the original $\mathcal{N}=6$ ABJM theory, the gauge group is $\mathrm{U}\left(N_{c}\right) \times \mathrm{U}\left(N_{c}\right)$ and there are matters in the bi-fundamental representation of the gauge group. We can add matters in the (anti-)fundamental representation of either $\mathrm{U}\left(N_{c}\right)$ group. After adding flavors, the maximal supersymmetry one can achieve is three-dimensional $\mathcal{N}=3$ supersymmetry [1012]. There was speculation that flavored ABJM theory should also be integrable [13], however no progress has been reported in this direction. In this paper, we will fill the gap and establish the two-loop integrability of this theory in the scalar sector.

For gauge theory with fundamental matters, there are two choices one can make when the planar (large $N_{c}$ ) limit is taken. One is the 't Hooft limit in which we let the number of flavors $N_{f}$ fixed. In this case, the contributions from Feynman diagrams involving fundamental matter loops will be suppressed. Another choice is the Veneziano limit in which we let $N_{f}$ go to infinity as well and keep the ratio $N_{f} / N_{c}$ finite. In this case, one should also include the planar Feynman diagrams involving fundamental matter loops. In this paper, we will work in the 't Hooft limit. This limit will simplify our computation greatly comparing with the Veneziano limit.

As in four dimensional cases, there are two types of gauge invariant composite operators one can consider in the scalar sector of flavored ABJM theory. The operator of the first type is built with bi-fundamental fields only. It is just the trace of product of bi-fundamental scalars placed alternatingly in the $\left(N_{c}, \bar{N}_{c}\right)$ and $\left(\bar{N}_{c}, N_{c}\right)$ representations of the gauge group. These operators are also the ones which appear in the scalar sector of ABJM theory and can be mapped to states of an alternating closed spin chain. In the 't Hooft limit, the computation of ADM of these operators is exactly the same as the one in ABJM theory, so we no longer need to repeat the study here. This type of operator will be called 'single trace operator'. The second type of gauge invariant operators will involve (anti-)fundamental scalars at two ends besides the bi-fundamental ones in the bulk. These operators will be called 'mesonic operators' and they can be mapped to states of an open spin chain. The main task of this paper is to compute the two-loop ADM of these mesonic operators and show that the corresponding Hamiltonian of this spin chain is integrable. In the 't Hooft limit, the bulk part of the Hamiltonian is the same as the one in ABJM theory and thus we only need to perform two-loop computations to get the boundary part of the Hamiltonian which involves both nearest and next-to-nearest neighbour interactions. Among them, there are two-site trace operators which do not exist in the total bulk Hamiltonian. The boundary terms will break the original $\mathrm{SU}(4)_{R}$ symmetry of the bulk interaction into

\footnotetext{
${ }^{1}$ Here and the following, by 'bulk', we mean the bulk of the composite fields. We hope this will not cause any confusion with the meaning of the 'bulk' in the holographic gauge/gravity duality.
} 
$\mathrm{SU}(2)_{R} \times \mathrm{SU}(2)_{D}$. We tried a lot to prove or disprove the integrability of the Hamiltonian based on algebraic Bethe ansatz, but we have not been successful yet.

This led us turn to the coordinate Bethe ansatz. In the context of AdS/CFT integrability, the coordinate Bethe ansatz method has been applied in [14] to show the integrability of an open spin chain model from giant gravitons. In this approach and for open chain, one should compute the bulk S-matrix and the reflection matrix (boundary S-matrix) and in order to show the integrability, one should check whether the Yang-Baxter equation (YBE) and the reflection equation are satisfied. The bulk S-matrix is the same as the one in ABJM theory which has already been computed in [15] to check the correctness of the all-order S-matrix proposed in [16]. We confirmed that YBE is satisfied by this S-matrix. As for the boundary reflection, we notice that it mixes magnons of different types and this is quite different from the case in four-dimensional SYM with fundamental matters [7, 17] where the boundary reflection is diagonal. By solving the eigenvalue problem of the total Hamiltonian in the one-magnon sector based on coordinate Bethe ansatz, we find the boundary reflection matrix. Finally by verifying the reflection equations, we confirm that the flavored ABJM theory is indeed integrable.

The paper is organized as follows. In the next section, we will review the action of $\mathcal{N}=3$ flavored ABJM theory and re-write it into a manifestly $\mathrm{SU}(2)_{R}$ invariant form. Section 3 is devoted to the computation of the boundary part of the two-loop Hamiltonian. Reflection matrix is computed in section 4 and integrability is proved in this section as well. We will discuss some further directions in the final section of the main text. Three appendices are included to provide some technical details.

\section{The action of $\mathcal{N}=3$ flavored ABJM theory}

In this section, we will study a variation of original $\mathcal{N}=6$ ABJM theory by adding some fundamental flavors which has been proposed in [10-12]. As discussed in these papers, we focus on $\mathcal{N}=3$ case which has maximal supersymmetry after the flavors are added. We will re-write the action into a manifestly $\mathrm{SU}(2)_{R} \sim \mathrm{SO}(3)_{R}$ invariant manner by the complete construction of the action in component fields including the fermionic part which is absent in the former investigation [10].

\subsection{The action in $\mathcal{N}=2$ superfield formulation}

The flavored ABJM theory has the product gauge group $\mathrm{U}\left(N_{c}\right) \times \mathrm{U}\left(N_{c}\right)$ with the ChernSimons levels $k$ and $-k$, respectively. The field content can be explicitly classified according to different representations of the gauge group. There are two hypermultiplets $\mathcal{Z}^{A}, A=1,2$, and $\mathcal{W}_{B}, B=1,2$, in bifundamental representations and two gauge multiplets $\mathcal{V}$ and $\hat{\mathcal{V}}$ in adjoint representations,

$$
\mathcal{Z}^{A} \in\left(N_{c}, \bar{N}_{c}\right), \quad \mathcal{W}_{A} \in\left(\bar{N}_{c}, N_{c}\right), \quad \mathcal{V} \in(\operatorname{adj}, 1), \quad \hat{\mathcal{V}} \in(1, \operatorname{adj})
$$


There are four kinds of flavors introduced by hypermultiplets belong to fundamental or anti-fundamental representations of each gauge group

$$
\begin{array}{lll}
b_{t} \in\left(1, N_{c}\right), & a^{t} \in\left(1, \bar{N}_{c}\right), & t=1, \cdots, N_{f_{1}}, \\
c^{s} \in\left(N_{c}, 1\right), & d_{s} \in\left(\bar{N}_{c}, 1\right), & s=1, \cdots, N_{f_{2}},
\end{array}
$$

with arbitrary number of $N_{f_{1}}$ and $N_{f_{2}}$.

The total action $\mathcal{S}=\mathcal{S}_{\mathrm{CS}}+\mathcal{S}_{\text {mat }}+\mathcal{S}_{\text {pot }}$ in $\mathcal{N}=2$ superspace can be formulated as the sum of the following three parts:

- Chern-Simons part

$$
\mathcal{S}_{\mathrm{CS}}=-\frac{i k}{8 \pi} \int d^{3} x d^{4} \theta \int_{0}^{1} d t \operatorname{tr}\left[\mathcal{V} \bar{D}^{\alpha}\left(e^{t \mathcal{V}} D_{\alpha} e^{-t \mathcal{V}}\right)-\hat{\mathcal{V}} \bar{D}^{\alpha}\left(e^{t \hat{\mathcal{V}}} D_{\alpha} e^{-t \hat{\mathcal{V}}}\right)\right],
$$

where the supercovariant derivatives are

$$
D_{\alpha}=\partial_{\alpha}+i\left(\gamma^{\mu} \bar{\theta}\right)_{\alpha} \partial_{\mu}, \quad \bar{D}_{\alpha}=-\bar{\partial}_{\alpha}-i\left(\theta \gamma^{\mu}\right)_{\alpha} \partial_{\mu} .
$$

- Matter part

$$
\begin{aligned}
\mathcal{S}_{\text {mat }}= & \int d^{3} x d^{4} \theta \operatorname{tr}\left(-\overline{\mathcal{Z}}_{A} e^{-\mathcal{V}} \mathcal{Z}^{A} e^{\hat{\mathcal{V}}}-\overline{\mathcal{W}}^{A} e^{-\hat{\mathcal{V}}} \mathcal{W}_{A} e^{\mathcal{V}}\right) \\
& +\int d^{3} x d^{4} \theta\left(-\bar{c}_{s} e^{-\mathcal{V}} c^{s}-\bar{b}^{t} e^{-\hat{\mathcal{V}}_{b}} b_{t}-d_{s} e^{\mathcal{V}} \bar{d}^{s}-a^{t} e^{\hat{\mathcal{V}}_{a_{t}}}\right)
\end{aligned}
$$

- Superpotential part

$$
\mathcal{S}_{\text {pot }}=\int d^{3} x d^{2} \theta \mathbb{W}(\mathcal{Z}, \mathcal{W}, c, d, b, a)+\text { c.c. },
$$

with the superpotential

$$
\mathbb{W}=-\frac{2 \pi}{k} \operatorname{tr}\left(\mathcal{Z}^{A} \mathcal{W}_{A}+c^{s} d_{s}\right)^{2}+\frac{2 \pi}{k} \operatorname{tr}\left(\mathcal{W}_{A} \mathcal{Z}^{A}+b_{t} a^{t}\right)^{2}
$$

\subsection{The action in $\mathcal{N}=2$ component field formulation}

The component expansions of our superfields are ${ }^{2}$

$$
\begin{aligned}
& a^{t}\left(x_{L}, \theta\right)=A^{t}+\sqrt{2} \theta \kappa^{t}+\theta^{2} I^{t}, \quad \bar{a}_{t}\left(x_{R}, \bar{\theta}\right)=A_{t}^{\dagger}-\sqrt{2} \bar{\theta} \kappa_{t}^{\dagger}-\bar{\theta}^{2} I_{t}^{\dagger}, \\
& b_{t}\left(x_{L}, \theta\right)=B_{t}+\sqrt{2} \theta \eta_{t}+\theta^{2} H_{t}, \quad \bar{b}^{t}\left(x_{R}, \bar{\theta}\right)=B^{\dagger t}-\sqrt{2} \bar{\theta} \eta^{\dagger t}-\bar{\theta}^{2} H^{\dagger t}, \\
& c^{s}\left(x_{L}, \theta\right)=C^{s}+\sqrt{2} \theta \tau^{s}+\theta^{2} J^{s}, \quad \bar{c}_{s}\left(x_{R}, \bar{\theta}\right)=C_{s}^{\dagger}-\sqrt{2} \bar{\theta} \tau_{s}^{\dagger}-\bar{\theta}^{2} J_{s}^{\dagger}, \\
& d_{s}\left(x_{L}, \theta\right)=E_{s}+\sqrt{2} \theta v_{s}+\theta^{2} K_{s}, \quad \bar{d}^{s}\left(x_{R}, \bar{\theta}\right)=E^{\dagger s}-\sqrt{2} \bar{\theta} v^{\dagger s}-\bar{\theta}^{2} K^{\dagger s}, \\
& \mathcal{Z}^{A}\left(x_{L}, \theta\right)=Z^{A}+\sqrt{2} \theta \zeta^{A}+\theta^{2} F^{A}, \quad \overline{\mathcal{Z}}_{A}\left(x_{R}, \bar{\theta}\right)=Z_{A}^{\dagger}-\sqrt{2} \bar{\theta} \zeta_{A}^{\dagger}-\bar{\theta}^{2} F_{A}^{\dagger}, \\
& \mathcal{W}_{A}\left(x_{L}, \theta\right)=W_{A}+\sqrt{2} \theta \omega_{A}+\theta^{2} G_{A}, \quad \overline{\mathcal{W}}^{A}\left(x_{R}, \bar{\theta}\right)=W^{\dagger A}-\sqrt{2} \bar{\theta} \omega^{\dagger A}-\bar{\theta}^{2} G^{\dagger A}, \\
& \mathcal{V}=2 i \theta \bar{\theta} \sigma(x)+2 \theta \gamma^{\mu} \bar{\theta} A_{\mu}(x)+\sqrt{2} i \theta^{2} \bar{\theta} \bar{\chi}(x)-\sqrt{2} i \bar{\theta}^{2} \theta \chi(x)+\theta^{2} \bar{\theta}^{2} D(x), \\
& \hat{\mathcal{V}}=2 i \theta \bar{\theta} \hat{\sigma}(x)+2 \theta \gamma^{\mu} \bar{\theta} \hat{A}_{\mu}(x)+\sqrt{2} i \theta^{2} \bar{\theta} \hat{\bar{\chi}}(x)-\sqrt{2} i \bar{\theta}^{2} \theta \hat{\chi}(x)+\theta^{2} \bar{\theta}^{2} \hat{D}(x) .
\end{aligned}
$$

Notice that the expansions of the vector superfields are in Wess-Zumino gauge.

\footnotetext{
${ }^{2}$ We follow the convention in [18].
} 
Following the treatment of deriving the component form of ABJM action [18], we integrate out those auxiliary fields and then we find the total action becomes,

$$
\begin{aligned}
\mathcal{S}_{\mathcal{N}=2}= & \int d^{3} x\left(\frac{k}{4 \pi} \operatorname{tr} \epsilon^{\mu \nu \lambda}\left(A_{\mu} \partial_{\nu} A_{\lambda}+\frac{2 i}{3} A_{\mu} A_{\nu} A_{\lambda}-\hat{A}_{\mu} \partial_{\nu} \hat{A}_{\lambda}-\frac{2 i}{3} \hat{A}_{\mu} \hat{A}_{\nu} \hat{A}_{\lambda}\right)\right. \\
& -\operatorname{tr}\left(\mathcal{D}^{\mu} Z\right)_{A}^{\dagger} \mathcal{D}_{\mu} Z^{A}-\operatorname{tr}\left(\mathcal{D}^{\mu} W\right)^{\dagger A} \mathcal{D}_{\mu} W_{A}-\operatorname{tr}\left(\mathcal{D}^{\mu} C\right)_{s}^{\dagger} \mathcal{D}_{\mu} C^{s}-\operatorname{tr}\left(\mathcal{D}^{\mu} B\right)^{\dagger t} \mathcal{D}_{\mu} B_{t} \\
& -\operatorname{tr}\left(\mathcal{D}^{\mu} E\right)^{\dagger s} \mathcal{D}_{\mu} E_{s}-\operatorname{tr}\left(\mathcal{D}^{\mu} A\right)_{t}^{\dagger} \mathcal{D}_{\mu} A^{t}-i \operatorname{tr} \zeta_{A}^{\dagger} \mathcal{D} \zeta^{A}-i \operatorname{tr} \omega^{\dagger A} \mathcal{D} \omega_{A}-i \operatorname{tr} \tau_{s}^{\dagger} \mathcal{D} \tau^{s} \\
& \left.-i \operatorname{tr} \eta^{\dagger t} \mathcal{D} \eta_{t}-i \operatorname{tr} v^{\dagger s} \not{D} v_{s}-i \operatorname{tr} \kappa_{t}^{\dagger} \mathcal{D} \kappa^{t}-V_{F}^{\text {bos }}-V_{F}^{\text {ferm }}-V_{D}^{\text {bos }}-V_{D}^{\text {ferm }}\right),
\end{aligned}
$$

where the covariant derivatives are defined as,

$$
\begin{aligned}
& \mathcal{D}_{\mu} \Phi^{A}=\partial_{\mu} \Phi^{A}+i A_{\mu} \Phi^{A}-i \Phi^{A} \hat{A}_{\mu}, \quad \text { for } \quad \Phi^{A} \in\left(N_{c}, \bar{N}_{c}\right), \\
& \mathcal{D}_{\mu} \phi^{s}=\partial_{\mu} \phi^{s}+i A_{\mu} \phi^{s}, \quad \text { for } \quad \phi^{s} \in\left(N_{c}, 1\right) \text {, } \\
& \mathcal{D}_{\mu} \hat{\phi}_{t}=\partial_{\mu} \hat{\phi}_{t}+i \hat{A}_{\mu} \hat{\phi}_{t}, \quad \text { for } \quad \hat{\phi}_{t} \in\left(1, N_{c}\right) \text {. }
\end{aligned}
$$

We put the lengthy expressions of the potential terms in appendix A together with the on-shell values of auxiliary fields.

\subsection{The action in $\mathcal{N}=3$ component field formulation}

In order to obtain a manifestly $\mathrm{SU}(2)_{R}$ invariant theory, we combine the component fields into the following doublet form

$$
\begin{aligned}
& X^{a A}=\left(\begin{array}{l}
Z^{A} \\
W^{\dagger A}
\end{array}\right), \quad X_{a A}^{\dagger}=\left(\begin{array}{c}
Z_{A}^{\dagger} \\
W_{A}
\end{array}\right), \quad \xi^{a A}=\left(\begin{array}{c}
\omega^{\dagger A} e^{i \pi / 4} \\
\zeta^{A} e^{-i \pi / 4}
\end{array}\right), \quad \xi_{a A}^{\dagger}=\left(\begin{array}{l}
\omega_{A} e^{-i \pi / 4} \\
\zeta_{A}^{\dagger} e^{i \pi / 4}
\end{array}\right) \\
& Y^{a s}=\left(\begin{array}{c}
C^{s} \\
E^{\dagger s}
\end{array}\right), \quad Y_{a s}^{\dagger}=\left(\begin{array}{c}
C_{s}^{\dagger} \\
E_{s}
\end{array}\right), \quad \psi^{a s}=\left(\begin{array}{c}
v^{\dagger s} e^{i \pi / 4} \\
\tau^{s} e^{-i \pi / 4}
\end{array}\right), \quad \psi_{a s}^{\dagger}=\left(\begin{array}{c}
v_{s} e^{-i \pi / 4} \\
\tau_{s}^{\dagger} e^{i \pi / 4}
\end{array}\right), \\
& M^{a t}=\left(\begin{array}{c}
A^{t} \\
B^{\dagger t}
\end{array}\right), \quad M_{a t}^{\dagger}=\left(\begin{array}{c}
A_{t}^{\dagger} \\
B_{t}
\end{array}\right), \quad \theta^{a t}=\left(\begin{array}{c}
\eta^{\dagger t} e^{i \pi / 4} \\
\kappa^{t} e^{-i \pi / 4}
\end{array}\right), \quad \theta_{a t}^{\dagger}=\left(\begin{array}{c}
\eta_{t} e^{-i \pi / 4} \\
\kappa_{t}^{\dagger} e^{i \pi / 4}
\end{array}\right)
\end{aligned}
$$

where the explicit $\mathrm{SU}(2)_{R} \mathrm{R}$-symmetry index $a$ is raised and lowered by the anti-symmetric tensor $\epsilon^{a b}$ and $\epsilon_{a b}$ with $\epsilon^{12}=-\epsilon_{12}=1 .^{3}$

In light of the work in [19] where an $\mathcal{N}=3$ Chern-Simons Yang-Mills theory was given, we re-write the above action into a manifestly $\mathrm{SU}(2)_{R}$ invariant form in terms of these new

\footnotetext{
${ }^{3}$ In the following we will also use $i, j, \cdots$ as R-symmetry indices.
} 
fields as

$$
\begin{aligned}
\mathcal{S}_{\mathcal{N}=3}= & \int d^{3} x \operatorname{tr}\left[\frac{k}{4 \pi} \epsilon^{\mu \nu \lambda}\left(A_{\mu} \partial_{\nu} A_{\lambda}+\frac{2 i}{3} A_{\mu} A_{\nu} A_{\lambda}\right)-\frac{k}{4 \pi} \epsilon^{\mu \nu \lambda}\left(\hat{A}_{\mu} \partial_{\nu} \hat{A}_{\lambda}+\frac{2 i}{3} \hat{A}_{\mu} \hat{A}_{\nu} \hat{A}_{\lambda}\right)\right. \\
& -\mathcal{D}_{\mu} X_{a A}^{\dagger} \mathcal{D}^{\mu} X^{a A}-\mathcal{D}_{\mu} Y_{a}^{\dagger} \mathcal{D}^{\mu} Y^{a}-\mathcal{D}_{\mu} M_{a}^{\dagger} \mathcal{D}^{\mu} M^{a}+i \xi_{a A}^{\dagger} \mathcal{D} \xi^{a A}+i \psi_{a}^{\dagger} \not{D} \psi^{a}+i \theta_{a}^{\dagger} \mathcal{D} \theta^{a} \\
& \left.-V_{\text {ferm }}^{\mathcal{N}=3}-V_{\text {bos }}^{\mathcal{N}=3}\right],
\end{aligned}
$$

with the fermionic part of the potential ${ }^{4}$

$$
\begin{aligned}
& -V_{\text {ferm }}^{\mathcal{N}=3}= \\
& \quad-\frac{2 \pi i}{k} \epsilon_{b c} \epsilon_{a d}\left(\xi^{a A} X_{A}^{\dagger b}-X^{b A} \xi_{A}^{\dagger a}+\psi^{a} Y^{\dagger b}-Y^{b} \psi^{\dagger a}\right)\left(\xi^{c B} X_{B}^{\dagger d}-X^{d B} \xi_{B}^{\dagger c}+\psi^{c} Y^{\dagger d}-Y^{d} \psi^{\dagger c}\right) \\
& \quad+\frac{2 \pi i}{k} \epsilon_{b c} \epsilon_{a d}\left(-\xi_{A}^{\dagger a} X^{b A}+X_{A}^{\dagger b} \xi^{a A}-\theta^{\dagger a} M^{b}+M^{\dagger b} \theta^{a}\right)\left(-\xi_{B}^{\dagger c} X^{d B}+X_{B}^{\dagger d} \xi^{c B}-\theta^{\dagger c} M^{d}+M^{\dagger d} \theta^{c}\right) \\
& \quad+\frac{4 \pi i}{k} \epsilon_{a c}\left(\xi^{a A} \xi_{b A}^{\dagger}+\psi^{a} \psi_{b}^{\dagger}\right)\left(X^{(c|B|} X_{B}^{\dagger b)}+Y^{(c} Y^{\dagger b)}\right) \\
& \quad-\frac{4 \pi i}{k} \epsilon_{a c}\left(\xi_{b A}^{\dagger} \xi^{a A}+\theta_{b}^{\dagger} \theta^{a}\right)\left(X_{B}^{\dagger(c} X^{b) B}+M^{\dagger(c} M^{b)}\right),
\end{aligned}
$$

and the bosonic part, which is first given in $[10],{ }^{5}$

$$
\begin{aligned}
& -V_{\mathrm{bos}}^{\mathcal{N}=3}= \\
& \frac{4 \pi^{2}}{3 k^{2}}\left[Y^{a} Y_{a}^{\dagger} Y^{b} Y_{b}^{\dagger} Y^{c} Y_{c}^{\dagger}+M_{a}^{\dagger} M^{a} M_{b}^{\dagger} M^{b} M_{c}^{\dagger} M^{c}-4 Y^{a} Y_{b}^{\dagger} Y^{c} Y_{a}^{\dagger} Y^{b} Y_{c}^{\dagger}-4 M_{a}^{\dagger} M^{b} M_{c}^{\dagger} M^{a} M_{b}^{\dagger} M^{c}\right. \\
& +X^{a A} X_{a A}^{\dagger} X^{b B} X_{b B}^{\dagger} X^{c M} X_{c M}^{\dagger}+X_{a A}^{\dagger} X^{a A} X_{b B}^{\dagger} X^{b B} X_{c M}^{\dagger} X^{c M}+4 X^{a A} X_{b B}^{\dagger} X^{c M} X_{a A}^{\dagger} X^{b B} X_{c M}^{\dagger} \\
& -6 X^{a A} X_{b B}^{\dagger} X^{b B} X_{a A}^{\dagger} X^{c M} X_{c M}^{\dagger}+3 X^{a A} X_{a A}^{\dagger} X^{b B} X_{b B}^{\dagger} Y^{c} Y_{c}^{\dagger}+3 X_{a A}^{\dagger} X^{a A} X_{b B}^{\dagger} X^{b B} M_{c}^{\dagger} M^{c} \\
& -6 X^{a A} X_{b B}^{\dagger} X^{b B} X_{a A}^{\dagger} Y^{c} Y_{c}^{\dagger}-6 X_{a A}^{\dagger} X^{b B} X_{b B}^{\dagger} X^{a A} M_{c}^{\dagger} M^{c}+9 X^{a A} X_{a A}^{\dagger} Y^{b} Y_{b}^{\dagger} Y^{c} Y_{c}^{\dagger} \\
& +9 X_{a A}^{\dagger} X^{a A} M_{b}^{\dagger} M^{b} M_{c}^{\dagger} M^{c}-6 X^{a A} X_{a A}^{\dagger} Y^{b} Y_{c}^{\dagger} Y^{c} Y_{b}^{\dagger}-6 X_{a A}^{\dagger} X^{a A} M_{b}^{\dagger} M^{c} M_{c}^{\dagger} M^{b} \\
& -6 X^{a A} X_{b A}^{\dagger} Y^{b} Y_{a}^{\dagger} Y^{c} Y_{c}^{\dagger}-6 X_{a A}^{\dagger} X^{b A} M_{b}^{\dagger} M^{a} M_{c}^{\dagger} M^{c}+6 X^{a A} X_{b A}^{\dagger} Y^{b} Y_{c}^{\dagger} Y^{c} Y_{a}^{\dagger} \\
& +6 X_{a A}^{\dagger} X^{b A} M_{b}^{\dagger} M^{c} M_{c}^{\dagger} M^{a}-6 X^{a A} X_{b A}^{\dagger} Y^{c} Y_{a}^{\dagger} Y^{b} Y_{c}^{\dagger}-6 X_{a A}^{\dagger} X^{b A} M_{c}^{\dagger} M^{a} M_{b}^{\dagger} M^{c} \\
& -6 X^{a A} X_{b A}^{\dagger} Y^{c} Y_{c}^{\dagger} Y^{b} Y_{a}^{\dagger}-6 X_{a A}^{\dagger} X^{b A} M_{c}^{\dagger} M^{c} M_{b}^{\dagger} M^{a}-6 X_{a A}^{\dagger} Y^{b} Y_{b}^{\dagger} X^{a A} M_{c}^{\dagger} M^{c} \\
& +12 X_{a A}^{\dagger} Y^{b} Y_{c}^{\dagger} X^{a A} M_{b}^{\dagger} M^{c}+12 \epsilon_{A B} \epsilon^{M N} X^{c A} X_{b M}^{\dagger} X^{a B} X_{c N}^{\dagger} Y^{b} Y_{a}^{\dagger} \\
& \left.+12 \epsilon^{A B} \epsilon_{M N} X_{c A}^{\dagger} X^{b M} X_{a B}^{\dagger} X^{c N} M_{b}^{\dagger} M^{a}\right],
\end{aligned}
$$

where flavor indices are suppressed.

\footnotetext{
${ }^{4}$ Our convention for symmetrization is $f_{(a b)}=\frac{1}{2}\left(f_{a b}+f_{b a}\right)$ and $f_{(a|B| b)}=\frac{1}{2}\left(f_{a B b}+f_{b B a}\right)$.

${ }^{5}$ In fact, there is a mistake in eq. (A.4) of the paper [10]: the second and the fourth terms should be corrected as $\bar{q}_{2}^{a} q_{a}^{2} \bar{q}_{2}^{b} q_{b}^{2} \bar{q}_{2}^{c} q_{c}^{2}$ and $-4 \bar{q}_{2}^{a} q_{b}^{2} \bar{q}_{2}^{c} q_{a}^{2} \bar{q}_{2}^{b} q_{c}^{2}$, respectively.
} 
Thus, we demonstrate the enhancement of the R-symmetry to $\mathrm{SU}(2)_{R}$ by the explicit construction of the action. Besides the $\mathrm{SU}(2)_{R}$ symmetry, the theory also has $\mathrm{SU}(2)_{D}$ symmetry acting on the $A$ index of $X^{a A}$. The above action is the starting point of our perturbative calculations.

\section{Two-loop perturbative calculations and the Hamiltonian}

In this section, we will compute the ADM of gauge invariant composite operators. We will perform the calculations in the 't Hooft limt with $N_{c} \rightarrow \infty, k \rightarrow \infty$ while $\lambda \equiv$ $N_{c} / k, N_{f_{1}}, N_{f_{2}}$ fixed. ${ }^{6}$ Since the ADM of single trace operators is the same as the one in the ABJM theory, we only need to consider the mesonic operators. We focus on two types of mesonic operators, ${ }^{7}$

$$
\begin{aligned}
& \hat{O}=Y_{i}^{\dagger} X^{i_{1} A_{1}} X_{i_{2} A_{2}}^{\dagger} \cdots X^{i_{2 L-1} A_{2 L-1}} X_{i_{2 L} A_{2 L}}^{\dagger} Y^{i^{\prime}} \\
& \hat{O}^{\prime}=Y_{i}^{\dagger} X^{i_{1} A_{1}} X_{i_{2} A_{2}}^{\dagger} \cdots X^{i_{2 L-1} A_{2 L-1}} X_{i_{2 L} A_{2 L}}^{\dagger} X^{i_{2 L+1} A_{2 L+1}} M_{i^{\prime}}^{\dagger}
\end{aligned}
$$

where $L>2$ and the contraction of the color indices is implied. We note that these composite operators are built up without trace operations since they are bounded on both sides by (anti-) fundamental matters. Our aim is to extract the ADM from the two point correlation function through 2-loop Feynman diagram computations. The calculations concerning only the bi-fundamental fields in the bulk are the same as those for the single trace operator $\operatorname{tr}\left(X X^{\dagger} \cdots X X^{\dagger}\right)$ in ABJM theory and have been carried out carefully in [5, $6] .{ }^{8}$ Here we will concentrate on the boundary part and show the details of the derivation of $\mathrm{ADM}$ of $\hat{O}$. For the operator $\hat{O}^{\prime}$, the whole procedure is identical and we will give the result directly in the end.

\subsection{Boundary three-site scalar interactions}

First we compute the contribution of the six-point vertex on the left boundary shown in figure 1. The relevant interaction terms in the $\mathcal{N}=3$ Lagrangian are:

$$
\begin{aligned}
V_{B}^{1} & =\frac{4 \pi^{2}}{k^{2}} X^{a A} X_{a A}^{\dagger} X^{b B} X_{b B}^{\dagger} Y^{c} Y_{c}^{\dagger}, \\
V_{B}^{2} & =-\frac{8 \pi^{2}}{k^{2}} X^{a A} X_{b B}^{\dagger} X^{b B} X_{a A}^{\dagger} Y^{c} Y_{c}^{\dagger}, \\
V_{B}^{3} & =\frac{16 \pi^{2}}{k^{2}} \epsilon_{A B} \epsilon^{M N} X^{c A} X_{b M}^{\dagger} X^{a B} X_{c N}^{\dagger} Y^{b} Y_{a}^{\dagger} .
\end{aligned}
$$

Let us analyze these interaction vertices separately and mainly focus on the flavor structure as follows:

\footnotetext{
${ }^{6}$ We will further set $N_{f_{1}}=N_{f_{2}}=1$ without loss of generality and then neglect the flavor indices for simplicity.

${ }^{7}$ There exist two other types of composite operators sharing essentially the same structures as those considered in the main text, namely, $M X^{\dagger} X \cdots X^{\dagger} X M^{\dagger}$ and $M X^{\dagger} X \cdots X^{\dagger} X X^{\dagger} Y$, and we will not repeat the similar analysis here.

${ }^{8}$ In $\beta$ - and $\gamma$-deformed ABJM theories, similar calculations have been performed in [20, 21].
} 


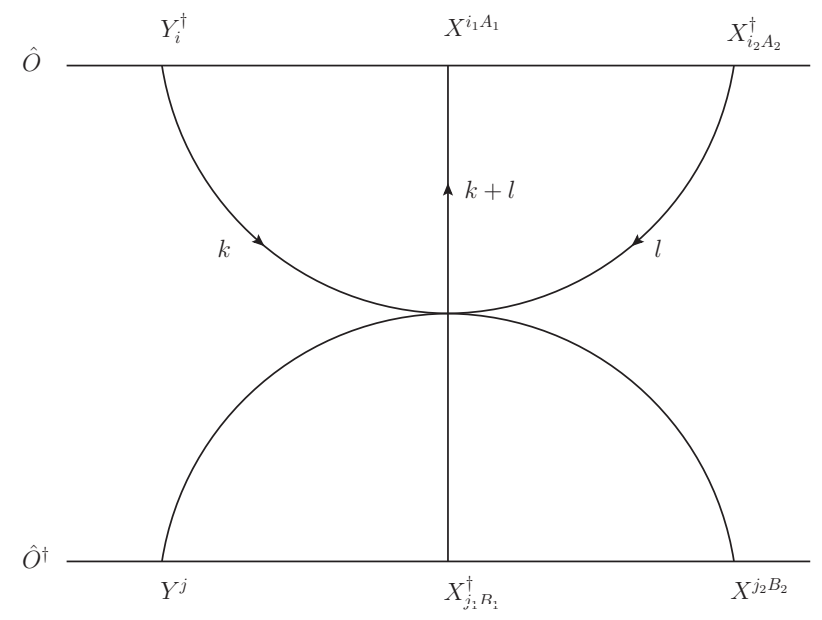

Figure 1. The contribution of six scalar interaction vertex on the left boundary.

- $V_{B}^{1}$ :

$$
\delta_{i}^{c} \delta_{b}^{i_{1}} \delta_{B}^{A_{1}} \delta_{i_{2}}^{b} \delta_{A_{2}}^{B} \delta_{a}^{j_{2}} \delta_{A}^{B_{2}} \delta_{j_{1}}^{a} \delta_{B_{1}}^{A} \delta_{c}^{j}=\delta_{i}^{j} \delta_{i_{2}}^{i_{1}} \delta_{j_{1}}^{j_{2}} \cdot \delta_{A_{2}}^{A_{1}} \delta_{B_{1}}^{B_{2}}
$$

- $V_{B}^{2}$

$$
\delta_{c}^{j} \delta_{i}^{c} \delta_{a}^{i_{1}} \delta_{A}^{A_{1}} \delta_{i_{2}}^{b} \delta_{A_{2}}^{B} \delta_{b}^{j_{2}} \delta_{B}^{B_{2}} \delta_{j_{1}}^{a} \delta_{B_{1}}^{A}=\delta_{i}^{j} \delta_{j_{1}}^{i_{1}} \delta_{i_{2}}^{j_{2}} \cdot \delta_{B_{1}}^{A_{1}} \delta_{A_{2}}^{B_{2}}
$$

- $V_{B}^{3}$ :

$$
\delta_{a}^{j} \delta_{i}^{b} \delta_{c}^{i_{1}} \delta_{N}^{A_{1}} \delta_{i_{2}}^{a} \delta_{A_{2}}^{B} \delta_{b}^{j_{2}} \delta_{M}^{B_{2}} \delta_{j_{1}}^{c} \delta_{B_{1}}^{A} \epsilon_{A B} \epsilon^{M N}=-\delta_{i}^{j_{2}} \delta_{i_{2}}^{j} \delta_{j_{1}}^{i_{1}} \cdot\left(\delta_{B_{1}}^{B_{2}} \delta_{A_{2}}^{A_{1}}-\delta_{B_{1}}^{A_{1}} \delta_{A_{2}}^{B_{2}}\right)
$$

We will use dimensional regularization to isolate the divergence and set $d=3-\epsilon$ with the relation: $\epsilon^{-1}=\log \Lambda^{2}$ where $\Lambda$ is the momentum space cutoff. The two-loop integral in figure 1 reads

$$
(-i)^{3}[i] \cdot i^{2} \int \frac{d^{d} k}{(2 \pi)^{d}} \frac{d^{d} l}{(2 \pi)^{d}} \frac{1}{(k+l)^{2}} \frac{1}{k^{2}} \frac{1}{l^{2}}=\frac{1}{16 \pi^{2}} \log \Lambda
$$

where the factor $[i]$ comes from the six-point vertex and $(-i)^{3}$ comes from the scalar propagator. The rest part of the above formula is a loop integral evaluated in Euclidean space and the factor $i^{2}$ accounts for the Wick rotation. There is also a factor $N_{c}^{2}$ from the contraction of color indices. Putting these together and noting that the contribution to the operator renormalization is negative of the quantum correction, we find the left boundary three-site scalar interaction gives

$$
\left(\mathcal{H}_{l}^{B}\right)_{i, j_{1} B_{1}, i_{2} A_{2}}^{j, i_{1} A_{1} j_{2} B_{2}}=-\frac{\lambda^{2}}{4}\left[\left(\delta_{i}^{j} \delta_{i_{2}}^{i_{1}} \delta_{j_{1}}^{j_{2}}-4 \delta_{i}^{j_{2}} \delta_{i_{2}}^{j} \delta_{j_{1}}^{i_{1}}\right) \delta_{A_{2}}^{A_{1}} \delta_{B_{1}}^{B_{2}}-2\left(\delta_{i}^{j} \delta_{j_{1}}^{i_{1}} \delta_{i_{2}}^{j_{2}}-2 \delta_{i}^{j_{2}} \delta_{j_{1}}^{i_{1}} \delta_{i_{2}}^{j}\right) \delta_{B_{1}}^{A_{1}} \delta_{A_{2}}^{B_{2}}\right] .
$$




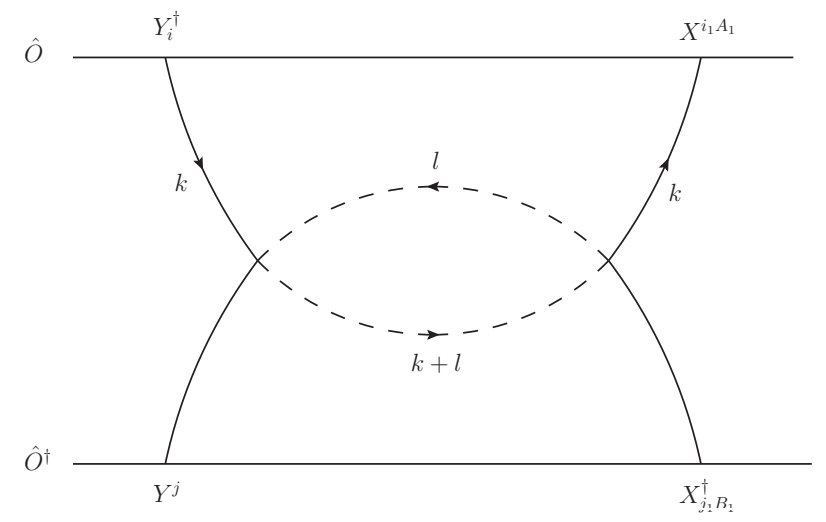

Figure 2. The contribution of Yukawa interactions on the left boundary.

The contribution from the right boundary can be obtained simply by some replacements of indices and we get

$$
\begin{aligned}
\left(\mathcal{H}_{r}^{B}\right)_{j_{2 L-1} B_{2 L-1}, i_{2 L} A_{2 L}, j^{\prime}}^{i_{2 L-1} A_{2 L-1}, j_{2 L} B_{2 L}, i^{\prime}}= & -\frac{\lambda^{2}}{4}\left[\left(\delta_{j^{\prime}}^{i^{\prime}} \delta_{j_{2 L-1}}^{j_{2 L}} \delta_{i_{2 L}}^{i_{2 L-1}}-4 \delta_{j^{\prime}}^{i_{2 L-1}} \delta_{j_{2 L-1}}^{i^{\prime}} \delta_{i_{2 L}}^{j_{2 L}}\right) \delta_{B_{2 L-1}}^{B_{2 L}} \delta_{A_{2 L}}^{A_{2 L-1}}\right. \\
& \left.-2\left(\delta_{j^{\prime}}^{i^{\prime}} j_{i_{2 L}}^{j_{2 L}} \delta_{j_{2 L-1}}^{i_{2 L-1}}-2 \delta_{j^{\prime}}^{i_{2 L-1}} \delta_{i_{2 L}}^{j_{2 L}} \delta_{j_{2 L-1}}^{i^{\prime}}\right) \delta_{A_{2 L}}^{B_{2 L}} \delta_{B_{2 L-1}}^{A_{2 L-1}}\right]
\end{aligned}
$$

\subsection{Boundary two-site Yukawa type interactions}

The Feynman diagram of the boundary two-site contribution consists of two Yukawa type vertices and a fermion loop depicted in figure 2. The involved interaction vertices are listed below:

$$
\begin{aligned}
V_{F}^{1} & =\frac{4 \pi i}{k} X^{a B} X_{A}^{\dagger b} \xi_{a}^{A} \xi_{b B}^{\dagger}, \\
V_{F}^{2} & =-\frac{2 \pi i}{k} X^{a B} X_{B}^{\dagger b} \xi_{a}^{A} \xi_{b A}^{\dagger}, \\
V_{F}^{3} & =-\frac{2 \pi i}{k} X^{a B} X_{B}^{\dagger b} \xi_{b}^{A} \xi_{a A}^{\dagger}, \\
\tilde{V}_{F}^{1} & =-\frac{2 \pi i}{k} Y^{a} Y^{\dagger b} \xi_{a}^{A} \xi_{b A}^{\dagger}, \\
\tilde{V}_{F}^{2} & =-\frac{2 \pi i}{k} Y^{a} Y^{\dagger b} \xi_{b}^{A} \xi_{a A}^{\dagger} .
\end{aligned}
$$

We have ignored the diagrams whose internal fermions belong to the fundamental flavors because such diagrams will be generically suppressed by a factor of $N_{f} / N_{c}$ in the 't Hooft limit. Now let us investigate the flavor structure arising from all possible combinations of the above vertices.

- $V_{F}^{1} \otimes \tilde{V}_{F}^{1}$ :

$$
\delta_{i}^{a} \epsilon_{m b} \epsilon_{a n} \epsilon^{n i_{1}} \delta_{j_{1}}^{m} \epsilon^{b j} \cdot \delta_{A}^{Q} \delta_{P}^{A} \delta_{Q}^{A_{1}} \delta_{B_{1}}^{P}=\delta_{i}^{i_{1}} \delta_{j_{1}}^{j} \delta_{B_{1}}^{A_{1}}
$$


- $V_{F}^{1} \otimes \tilde{V}_{F}^{2}$ :

$$
\delta_{i}^{a} \epsilon_{m a} \epsilon_{b n} \epsilon^{n i_{1}} \delta_{j_{1}}^{m} \epsilon^{b j} \cdot \delta_{A}^{Q} \delta_{P}^{A} \delta_{Q}^{A_{1}} \delta_{B_{1}}^{P}=\left(-\delta_{j_{1}}^{i_{1}} \delta_{i}^{j}+\delta_{j_{1}}^{j} \delta_{i}^{i_{1}}\right) \delta_{B_{1}}^{A_{1}},
$$

- $V_{F}^{2} \otimes \tilde{V}_{F}^{1}$

$$
\delta_{i}^{a} \epsilon_{m b} \epsilon_{a n} \epsilon^{n i_{1}} \delta_{j_{1}}^{m} \epsilon^{b j} \cdot \delta_{A}^{Q} \delta_{Q}^{A} \delta_{P}^{A_{1}} \delta_{B_{1}}^{P}=2 \delta_{j_{1}}^{j} \delta_{i}^{i_{1}} \delta_{B_{1}}^{A_{1}},
$$

- $V_{F}^{2} \otimes \tilde{V}_{F}^{2}$ :

$$
\delta_{i}^{a} \epsilon_{m a} \epsilon_{b n} \epsilon^{n i_{1}} \delta_{j_{1}}^{m} \epsilon^{b j} \cdot \delta_{A}^{Q} \delta_{Q}^{A} \delta_{P}^{A_{1}} \delta_{B_{1}}^{P}=2\left(-\delta_{j_{1}}^{i_{1}} \delta_{i}^{j}+\delta_{j_{1}}^{j} \delta_{i}^{i_{1}}\right) \delta_{B_{1}}^{A_{1}},
$$

- $V_{F}^{3} \otimes \tilde{V}_{F}^{1}$ :

$$
\delta_{i}^{a} \epsilon_{n b} \epsilon_{a m} \epsilon^{n i_{1}} \delta_{j_{1}}^{m} \epsilon^{b j} \cdot \delta_{A}^{Q} \delta_{Q}^{A} \delta_{P}^{A_{1}} \delta_{B_{1}}^{P}=2\left(-\delta_{i}^{j} \delta_{j_{1}}^{i_{1}}+\delta_{i}^{i_{1}} \delta_{j_{1}}^{j}\right) \delta_{B_{1}}^{A_{1}}
$$

- $V_{F}^{3} \otimes \tilde{V}_{F}^{2}$ :

$$
\delta_{i}^{a} \epsilon_{n a} \epsilon_{b m} \epsilon^{n i_{1}} \delta_{j_{1}}^{m} \epsilon^{b j} \cdot \delta_{A}^{Q} \delta_{Q}^{A} \delta_{P}^{A_{1}} \delta_{B_{1}}^{P}=2 \delta_{i}^{i_{1}} \delta_{j_{1}}^{j} \delta_{B_{1}}^{A_{1}} .
$$

The remaining loop integral is

$$
\frac{2}{2 !}(-i)^{2}(i)^{2}[i]^{2} \cdot(-1) i^{2} \int \frac{d^{d} k}{(2 \pi)^{d}} \frac{d^{d} l}{(2 \pi)^{d}} \frac{1}{\left(k^{2}\right)^{2}} \operatorname{tr}\left(\frac{\gamma^{\mu} l_{\mu}}{l^{2}} \frac{\gamma^{\nu}(k+l)_{\nu}}{(k+l)^{2}}\right)=\frac{1}{16 \pi^{2}} \log \Lambda,
$$

where the factor $2 / 2$ ! is from the coefficient of the second order expansion of the interaction terms and $(-i)^{2},(i)^{2},[i]^{2}$ come from the scalar and fermion propagators and the vertices respectively. The factor $(-1)$ is from fermion loop accounting for the Fermi-Dirac statistics. Gathering all these data, we find the final counter-term contributing to the dilatation operator is

$$
\left(\mathcal{H}_{l}^{F}\right)_{i, j_{1} B_{1}, i_{2} A_{2}}^{j, i_{1} A_{1}, j_{2} B_{2}}=\frac{\lambda^{2}}{2}\left(2 \delta_{i}^{i_{1}} \delta_{j_{1}}^{j}-\delta_{i}^{j} \delta_{j_{1}}^{i_{1}}\right) \delta_{i_{2}}^{j_{2}} \delta_{B_{1}}^{A_{1}} \delta_{A_{2}}^{B_{2}} .
$$

For the right boundary, it is

$$
\left(\mathcal{H}_{r}^{F}\right)_{j_{2 L-1} B_{2 L-1}, i_{2 L} A_{2 L}, j^{\prime}}^{i_{2 L-1} A_{2 L-1}, j_{2 L} B_{2 L}, i^{\prime}}=\frac{\lambda^{2}}{2}\left(2 \delta_{j^{\prime}}^{j_{2 L}} \delta_{i_{2 L}}^{i^{\prime}}-\delta_{j^{\prime}}^{i^{\prime}} \delta_{i_{2 L}}^{j_{2 L}}\right) \delta_{j_{2 L-1}}^{i_{2 L-1}} \delta_{A_{2 L}}^{B_{2 L}} \delta_{B_{2 L-1}}^{A_{2 L-1}}
$$

\subsection{The two-loop Hamiltonian}

There is another two-site diagram concerning the exchange interaction of gauge bosons, however, this Feynman diagram can only give constant contribution since the gauge propagators do not carry flavor indices. As for the various one-site diagrams representing the wave function renormalization, it is easy to see that they also lead to constant pieces. Note also that the two two-bulk-site trace operators in $\mathcal{H}_{l}^{B}$ and $\mathcal{H}_{r}^{B}$ are canceled by the bulk two-site interactions. And this cancelation makes the whole bulk Hamiltonian to be in the 
same form as the one derived from the single trace operator in ABJM theory. Finally, the two-loop Hamiltonian associated with the ADM of the composite operator $\hat{O}$ reads

$$
\mathcal{H}=\mathcal{H}_{l}+\mathcal{H}_{r}+\mathcal{H}_{\text {bulk }}+\alpha \mathbb{I}
$$

with

$$
\begin{aligned}
& \left(\mathcal{H}_{l}\right)_{i, j_{1} B_{1}, i_{2} A_{2}}^{j, i_{1} A_{1} j_{2} B_{2}}=\lambda^{2}\left[\left(\delta_{A_{2}}^{A_{1}} \delta_{B_{1}}^{B_{2}}-\delta_{B_{1}}^{A_{1}} \delta_{A_{2}}^{B_{2}}\right) \cdot \delta_{i}^{j_{2}} \delta_{j_{1}}^{i_{1}} \delta_{i_{2}}^{j}+\delta_{B_{1}}^{A_{1}} \delta_{A_{2}}^{B_{2}} \cdot \delta_{i}^{i_{1}} \delta_{j_{1}}^{j} \delta_{i_{2}}^{j_{2}}\right], \\
& \left(\mathcal{H}_{r}\right)_{j_{2 L-1} B_{2 L-1}, i_{2 L} A_{2 L}, j^{\prime}}^{i_{2 L-1} A_{2 L-1}, j_{2 L} B_{2 L}, i^{\prime}}=\lambda^{2}\left[\left(\delta_{B_{2 L-1}}^{B_{2 L}} \delta_{A_{2 L}}^{A_{2 L-1}}-\delta_{A_{2 L}}^{B_{2 L}} \delta_{B_{2 L-1}}^{A_{2 L-1}}\right) \cdot \delta_{j^{\prime}}^{i_{2 L-1}} \delta_{i_{2 L}}^{j_{2 L}} \delta_{j_{2 L-1}}^{i^{\prime}}\right. \\
& \left.+\delta_{A_{2 L}}^{B_{2 L}} \delta_{B_{2 L-1}}^{A_{2 L-1}} \cdot \delta_{j_{2 L-1}}^{i_{2 L-1}} \delta_{i_{2 L}}^{i^{\prime}} \delta_{j^{\prime}}^{j_{2 L}}\right], \\
& \mathcal{H}_{\text {bulk }}=\lambda^{2} \sum_{l=1}^{2 L-2}\left(\mathbb{I}_{l, l+1}-\mathbb{P}_{l, l+2}+\frac{1}{2} \mathbb{P}_{l, l+2} \mathbb{K}_{l, l+1}+\frac{1}{2} \mathbb{K}_{l, l+1} \mathbb{P}_{l, l+2}\right),
\end{aligned}
$$

where the basic operators $\mathbb{I}, \mathbb{P}$ and $\mathbb{K}$ are defined as

$$
\left(\mathbb{I}_{l, l+1}\right)_{j B, i^{\prime} A^{\prime}}^{i A, j^{\prime} B^{\prime}}=\delta_{j}^{i} \delta_{i^{\prime}}^{j^{\prime}} \delta_{B}^{A} \delta_{A^{\prime}}^{B^{\prime}}, \quad\left(\mathbb{P}_{l, l+2}\right)_{j B, j^{\prime} B^{\prime}}^{i A, i^{\prime} A^{\prime}}=\delta_{j^{\prime}}^{i} \delta_{j}^{i^{\prime}} \delta_{B^{\prime}}^{A} \delta_{B}^{A^{\prime}}, \quad\left(\mathbb{K}_{l, l+1}\right)_{j B, i^{\prime} A^{\prime}}^{i A, j^{\prime} B^{\prime}}=\delta_{i^{\prime}}^{i} \delta_{j}^{j^{\prime}} \delta_{A^{\prime}}^{A} \delta_{B}^{B^{\prime}},
$$

and the exact value of the coefficient $\alpha$ will be determined later by the BPS condition of the corresponding vacuum state. For operator $\hat{O}^{\prime}$, the two-loop Hamiltonian is

$$
\mathcal{H}^{\prime}=\mathcal{H}_{l}^{\prime}+\mathcal{H}_{r}^{\prime}+\mathcal{H}_{\mathrm{bulk}}^{\prime}+\alpha^{\prime} \mathbb{I}
$$

where

$$
\begin{aligned}
\mathcal{H}_{l}^{\prime} & =\mathcal{H}_{l}, \\
\left(\mathcal{H}_{r}^{\prime}\right)_{i_{2 L} A_{2 L}, j_{2 L+1} B_{2 L+1}, i^{\prime}}^{j_{2 L} B_{2 L}, i_{2 L+1} A_{2 L+1}, j^{\prime}} & =\lambda^{2}\left[\left(\delta_{A_{2 L}}^{A_{2 L+1}} \delta_{B_{2 L+1}}^{B_{2 L}}-\delta_{B_{2 L+1}}^{A_{2 L+1}} \delta_{A_{2 L}}^{B_{2 L}}\right) \cdot \delta_{i^{\prime}}^{j_{2 L}} \delta_{j_{2 L+1}}^{i_{2 L+1}} \delta_{i_{2 L}}^{j^{\prime}}\right. \\
& \left.+\delta_{B_{2 L+1}}^{A_{2 L+1}} \delta_{A_{2 L}}^{B_{2 L}} \cdot \delta_{i^{\prime}}^{i_{2 L+1}} \delta_{j_{2 L+1}}^{j^{\prime}} \delta_{i_{2 L}}^{j_{2 L}}\right] \\
\mathcal{H}_{\text {bulk }}^{\prime} & =\lambda^{2} \sum_{l=1}^{2 L-1}\left(\mathbb{I}_{l, l+1}-\mathbb{P}_{l, l+2}+\frac{1}{2} \mathbb{P}_{l, l+2} \mathbb{K}_{l, l+1}+\frac{1}{2} \mathbb{K}_{l, l+1} \mathbb{P}_{l, l+2}\right) .
\end{aligned}
$$

We would like to mention some features of the boundary interaction. It breaks the $\mathrm{SU}(4)_{R}$ symmetry of the bulk interaction into $\mathrm{SU}(2)_{R} \times \mathrm{SU}(2)_{D}$. It includes both nearest and next-to-nearest neighbour interactions, especially the two-site trace operators ${ }^{9}$ which do not appear in the bulk interaction.

\section{Integrability from coordinate Bethe ansatz}

In this section, we will prove the integrability of flavored ABJM model by showing that the boundary reflection matrices satisfy the reflection equations. These reflection matrices are

\footnotetext{
${ }^{9}$ These involve the boundary site and the nearest bulk site.
} 
obtained by the concrete constructions of Bethe ansatz solutions of the Hamiltonian. We begin with the composite operator $\hat{O}$ which naturally corresponds to an open spin chain state and the vacuum or the Bethe reference state is chosen to be

$$
|\Omega\rangle=\left|Y_{2}^{\dagger} X^{11} X_{22}^{\dagger} \cdots X^{11} X_{22}^{\dagger} Y^{1}\right\rangle
$$

For the case of single impurities, the Hamiltonian in eq. (3.26) reduces to

$$
\mathcal{H}=\mathcal{H}_{l}+\mathcal{H}_{r}+\alpha \mathbb{I}+\lambda^{2} \sum_{l=1}^{2 L-2}\left(\mathbb{I}_{l, l+1}-\mathbb{P}_{l, l+2}\right) .
$$

In appendix B, we will demonstrate that the vacuum is a BPS state, so its scaling dimension receives no quantum corrections. This determines $\alpha$ to be $2 \lambda^{2}$. We now use a conventional way to label the bulk fields as A and B types as follows, ${ }^{10}$

$$
X^{11}=A_{1}, \quad X^{12}=A_{2}, \quad X^{21}=B_{1}^{\dagger}, \quad X^{22}=B_{2}^{\dagger} .
$$

There are three types of one-particle excitations,

$$
\begin{array}{cl}
\text { bulk A type: } & Y_{2}^{\dagger}\left(A_{1} B_{2}\right) \cdots\left(A_{2} B_{2}\right) \cdots\left(A_{1} B_{2}\right) Y^{1}, \\
& Y_{2}^{\dagger}\left(A_{1} B_{2}\right) \cdots\left(B_{1}^{\dagger} B_{2}\right) \cdots\left(A_{1} B_{2}\right) Y^{1}, \\
\text { bulk B type: } & Y_{2}^{\dagger}\left(A_{1} B_{2}\right) \cdots\left(A_{1} A_{2}^{\dagger}\right) \cdots\left(A_{1} B_{2}\right) Y^{1}, \\
& Y_{2}^{\dagger}\left(A_{1} B_{2}\right) \cdots\left(A_{1} B_{1}\right) \cdots\left(A_{1} B_{2}\right) Y^{1}, \\
\text { boundary: } & Y_{1}^{\dagger}\left(A_{1} B_{2}\right) \cdots\left(A_{1} B_{2}\right) \cdots\left(A_{1} B_{2}\right) Y^{1}, \\
& Y_{2}^{\dagger}\left(A_{1} B_{2}\right) \cdots\left(A_{1} B_{2}\right) \cdots\left(A_{1} B_{2}\right) Y^{2} .
\end{array}
$$

After scattering at the boundary, these pseudo-particles will transform into each other. Under the action of $\mathcal{H}_{l}$,

$$
\begin{aligned}
\mathcal{H}_{l}|1\rangle_{A_{2}} & =\lambda^{2}|1\rangle_{B_{1}}, \\
\mathcal{H}_{l}|1\rangle_{B_{1}^{\dagger}} & =\lambda^{2}|l\rangle_{Y_{1}^{\dagger}}, \\
\mathcal{H}_{l}|1\rangle_{A_{2}^{\dagger}} & =-\lambda^{2}|l\rangle_{Y_{1}^{\dagger}}, \\
\mathcal{H}_{l}|1\rangle_{B_{1}} & =\lambda^{2}|1\rangle_{A_{2}}, \\
\mathcal{H}_{l}|l\rangle_{Y_{1}^{\dagger}} & =-\lambda^{2}|1\rangle_{A_{2}^{\dagger}}+\lambda^{2}|l\rangle_{Y_{1}^{\dagger}}+\lambda^{2}|1\rangle_{B_{1}^{\dagger}}, \\
\mathcal{H}_{l}|x\rangle & =-\lambda^{2}|x\rangle, \quad x \neq 1,
\end{aligned}
$$

and under the action of $\mathcal{H}_{r}$,

$$
\begin{aligned}
\mathcal{H}_{r}|L\rangle_{A_{2}} & =\lambda^{2}|L\rangle_{B_{1}}, \\
\mathcal{H}_{r}|L\rangle_{B_{1}^{\dagger}} & =-\lambda^{2}|r\rangle_{Y^{2}}, \\
\mathcal{H}_{r}|L\rangle_{A_{2}^{\dagger}} & =\lambda^{2}|r\rangle_{Y^{2}}, \\
\mathcal{H}_{r}|L\rangle_{B_{1}} & =\lambda^{2}|L\rangle_{A_{2}}, \\
\mathcal{H}_{r}|r\rangle_{Y^{2}} & =-\lambda^{2}|L\rangle_{B_{1}^{\dagger}}+\lambda^{2}|r\rangle_{Y^{2}}+\lambda^{2}|L\rangle_{A_{2}^{\dagger}}, \\
\mathcal{H}_{r}|x\rangle & =-\lambda^{2}|x\rangle, \quad x \neq L,
\end{aligned}
$$

\footnotetext{
${ }^{10}$ This convention is the same as the one in [15], though it is different from the one in section 2.
} 
where the spin chain is symbolised as $|l(1)(2) \cdots(x) \cdots(L) r\rangle$ with every site $(x)$ containing two fields. We use the excitation with its position to label the state and use $|x\rangle$ without subscript to denote any of $|x\rangle_{A_{2}},|x\rangle_{B_{1}},|x\rangle_{A_{2}^{\dagger}},|x\rangle_{B_{1}^{\dagger}}$. Here we see the novelty of our model where different states can mix and nontrivial boundary reflections will appear unlike those parallel studies in SYM with fundamental matters [7, 17]. Then we find that only the superposition of several different one-particle spin wave functions can be constructed as an eigenstate of the Hamiltonian and we can extract the boundary reflection matrix by solving the corresponding eigenvalue equations.

\subsection{Two-particle mixed sector}

We consider the superposition of the spin wave functions of particles $A_{2}$ and $B_{1}$ as follows,

$$
\left|\psi_{1}(k)\right\rangle=\sum_{x=1}^{L}\left(f(x)|x\rangle_{A_{2}}+g(x)|x\rangle_{B_{1}}\right),
$$

where the Bethe ansatz for the wave functions are

$$
\begin{aligned}
& f(x)=F e^{i k x}+\tilde{F} e^{-i k x}, \\
& g(x)=G e^{i k x}+\tilde{G} e^{-i k x} .
\end{aligned}
$$

Using our Hamiltonian, we find that

$$
\begin{aligned}
\mathcal{H} \sum_{x=1}^{L} f(x)|x\rangle_{A_{2}}= & \lambda^{2} f(1)|1\rangle_{B_{1}}+\lambda^{2} f(L)|L\rangle_{B_{1}}+2 \lambda^{2} f(1)|1\rangle_{A_{2}}+2 \lambda^{2} f(L)|L\rangle_{A_{2}} \\
& -\lambda^{2} f(1)|2\rangle_{A_{2}}-\lambda^{2} f(L)|L-1\rangle_{A_{2}} \\
& +\lambda^{2} \sum_{x=2}^{L-1} f(x)\left(2|x\rangle_{A_{2}}-|x-1\rangle_{A_{2}}-|x+1\rangle_{A_{2}}\right)
\end{aligned}
$$

and

$$
\begin{aligned}
\mathcal{H} \sum_{x=1}^{L} g(x)|x\rangle_{B_{1}}= & \lambda^{2} g(1)|1\rangle_{A_{2}}+\lambda^{2} g(L)|L\rangle_{A_{2}}+2 \lambda^{2} g(1)|1\rangle_{B_{1}}+2 \lambda^{2} g(L)|L\rangle_{B_{1}} \\
& -\lambda^{2} g(1)|2\rangle_{B_{1}}-\lambda^{2} g(L)|L-1\rangle_{B_{1}} \\
& +\lambda^{2} \sum_{x=2}^{L-1} g(x)\left(2|x\rangle_{B_{1}}-|x-1\rangle_{B_{1}}-|x+1\rangle_{B_{1}}\right)
\end{aligned}
$$

The eigenvalue equation $\mathcal{H}\left|\psi_{1}\right\rangle=E(k)\left|\psi_{1}\right\rangle$ gives:

- The bulk part $(x \neq 1, L)$,

$$
\begin{aligned}
2 \lambda^{2} f(x)-\lambda^{2} f(x+1)-\lambda^{2} f(x-1) & =E f(x), \\
2 \lambda^{2} g(x)-\lambda^{2} g(x+1)-\lambda^{2} g(x-1) & =E g(x) .
\end{aligned}
$$

Substituting eq. (4.23) and eq. (4.24) into the above equations, we have the following dispersion relation for the proposed spin wave,

$$
E(k)=2 \lambda^{2}-2 \lambda^{2} \cos k .
$$


- The left boundary part,

$$
\begin{aligned}
& \lambda^{2} f(1)+2 \lambda^{2} g(1)-\lambda^{2} g(2)=E g(1), \\
& 2 \lambda^{2} f(1)-\lambda^{2} f(2)+\lambda^{2} g(1)=E f(1) .
\end{aligned}
$$

Using eqs. (4.27) and (4.28), the above coupled equations become

$$
\begin{aligned}
& f(1)=-g(0), \\
& g(1)=-f(0) .
\end{aligned}
$$

By the plane wave expansions of eq. (4.23) and eq. (4.24), we find the relations

$$
\begin{aligned}
& F e^{i k}+\tilde{F} e^{-i k}+G+\tilde{G}=0, \\
& G e^{i k}+\tilde{G} e^{-i k}+F+\tilde{F}=0 .
\end{aligned}
$$

The solution is

$$
F=-e^{-i k} \tilde{G}, \quad G=-e^{-i k} \tilde{F} .
$$

We define the left reflection matrix $K_{l}(k)$ in this sector by

$$
\left(\begin{array}{c}
F \\
G
\end{array}\right) \equiv K_{l}(k)\left(\begin{array}{c}
\tilde{F} \\
\tilde{G}
\end{array}\right) .
$$

So from the above solution, we have,

$$
K_{l}(k)=\left(\begin{array}{cc}
0 & -e^{-i k} \\
-e^{-i k} & 0
\end{array}\right) .
$$

- The right boundary part,

$$
\begin{aligned}
& \lambda^{2} f(L)+2 \lambda^{2} g(L)-\lambda^{2} g(L-1)=E g(L), \\
& 2 \lambda^{2} f(L)-\lambda^{2} f(L-1)+\lambda^{2} g(L)=E f(L),
\end{aligned}
$$

which can be reduced to

$$
\begin{aligned}
& f(L)+g(L+1)=0, \\
& g(L)+f(L+1)=0 .
\end{aligned}
$$

This gives

$$
\begin{aligned}
& F e^{i k L}+\tilde{F} e^{-i k L}+G e^{i k(L+1)}+\tilde{G} e^{-i k(L+1)}=0, \\
& G e^{i k L}+\tilde{G} e^{-i k L}+F e^{i k(L+1)}+\tilde{F} e^{-i k(L+1)}=0 .
\end{aligned}
$$

Solving the above two equations, we get

$$
F=-e^{-2 i k L-i k} \tilde{G}, \quad G=-e^{-2 i k L-i k} \tilde{F} .
$$


Following [22], we define the right reflection matrix $K_{r}(k)$ in this sector by

$$
e^{2 i k L}\left(\begin{array}{c}
F \\
G
\end{array}\right) \equiv K_{r}(k)\left(\begin{array}{c}
\tilde{F} \\
\tilde{G}
\end{array}\right) \text {. }
$$

Then we get

$$
K_{r}(k)=\left(\begin{array}{cc}
0 & -e^{-i k} \\
-e^{-i k} & 0
\end{array}\right) .
$$

The consistency of eq. (4.36) and (4.45) gives

$$
e^{2 i k L}=1, \quad k=\frac{n \pi}{L}, \quad n \in \mathbb{Z} .
$$

This is the quantization conditions for our spin wave momenta $k$ as well as the Bethe equation for this two-particle mixed sector.

\subsection{Four-particle mixed sector}

Now we turn to another closed sector which consists of four excitations $B_{1}^{\dagger}, A_{2}^{\dagger}, Y_{1}^{\dagger}$ and $Y^{2}$. The spin wave takes the form

$$
\left|\psi_{2}(k)\right\rangle=\sum_{x=1}^{L} n(x)|x\rangle_{B_{1}^{\dagger}}+\sum_{x=1}^{L} h(x)|x\rangle_{A_{2}^{\dagger}}+\beta|l\rangle_{Y_{1}^{\dagger}}+\gamma|r\rangle_{Y^{2}},
$$

with

$$
\begin{aligned}
& n(x)=N e^{i k x}+\tilde{N} e^{-i k x}, \\
& h(x)=H e^{i k x}+\tilde{H} e^{-i k x} .
\end{aligned}
$$

The Hamiltonian acts on the above wave function as follows

$$
\begin{aligned}
\mathcal{H} \sum_{x=1}^{L} n(x)|x\rangle_{B_{1}^{\dagger}}= & \lambda^{2} n(1)|l\rangle_{Y_{1}^{\dagger}}-\lambda^{2} n(L)|r\rangle_{Y^{2}}+2 \lambda^{2} n(1)|1\rangle_{B_{1}^{\dagger}}+2 \lambda^{2} n(L)|L\rangle_{B_{1}^{\dagger}} \\
& -\lambda^{2} n(1)|2\rangle_{B_{1}^{\dagger}}-\lambda^{2} n(L)|L-1\rangle_{B_{1}^{\dagger}} \\
& +\lambda^{2} \sum_{x=2}^{L-1} n(x)\left(2|x\rangle_{B_{1}^{\dagger}}-|x-1\rangle_{B_{1}^{\dagger}}-|x+1\rangle_{B_{1}^{\dagger}}\right), \\
\mathcal{H} \sum_{x=1}^{L} h(x)|x\rangle_{A_{2}^{\dagger}}= & -\lambda^{2} h(1)|l\rangle_{Y_{1}^{\dagger}}+\lambda^{2} h(L)|r\rangle_{Y^{2}}+2 \lambda^{2} h(1)|1\rangle_{A_{2}^{\dagger}}+2 \lambda^{2} h(L)|L\rangle_{A_{2}^{\dagger}} \\
& -\lambda^{2} h(1)|2\rangle_{A_{2}^{\dagger}}-\lambda^{2} h(L)|L-1\rangle_{A_{2}^{\dagger}} \\
& +\lambda^{2} \sum_{x=2}^{L-1} h(x)\left(2|x\rangle_{A_{2}^{\dagger}}-|x-1\rangle_{A_{2}^{\dagger}}-|x+1\rangle_{A_{2}^{\dagger}}\right),
\end{aligned}
$$


and

$$
\begin{aligned}
\mathcal{H}|l\rangle_{Y_{1}^{\dagger}} & =2 \lambda^{2}|l\rangle_{Y_{1}^{\dagger}}-\lambda^{2}|1\rangle_{A_{2}^{\dagger}}+\lambda^{2}|1\rangle_{B_{1}^{\dagger}}, \\
\mathcal{H}|r\rangle_{Y^{2}} & =2 \lambda^{2}|r\rangle_{Y^{2}}-\lambda^{2}|L\rangle_{B_{1}^{\dagger}}+\lambda^{2}|L\rangle_{A_{2}^{\dagger}} .
\end{aligned}
$$

We demand the proposed spin wave function to be an energy eigenstate:

$$
\mathcal{H}\left|\psi_{2}(k)\right\rangle=E(k)\left|\psi_{2}(k)\right\rangle,
$$

which leads to the following relations:

- The bulk part $(x \neq 1, L)$,

$$
\begin{aligned}
& 2 \lambda^{2} n(x)-\lambda^{2} n(x+1)-\lambda^{2} n(x-1)=E n(x), \\
& 2 \lambda^{2} h(x)-\lambda^{2} h(x+1)-\lambda^{2} h(x-1)=E h(x),
\end{aligned}
$$

which give the same dispersion relation

$$
E(k)=2 \lambda^{2}-2 \lambda^{2} \cos k .
$$

- The left boundary part,

$$
\begin{aligned}
& 2 \lambda^{2} n(1)-\lambda^{2} n(2)+\lambda^{2} \beta=E n(1), \\
& 2 \lambda^{2} h(1)-\lambda^{2} h(2)-\lambda^{2} \beta=E h(1), \\
& \lambda^{2} n(1)-\lambda^{2} h(1)+2 \lambda^{2} \beta=E \beta .
\end{aligned}
$$

Plugging eqs. (4.57) and (4.58) into these equations, we readily have

$$
\begin{aligned}
\beta & =h(0), \\
n(1) & =-h(-1), \\
n(0) & =-h(0),
\end{aligned}
$$

which means

$$
\begin{aligned}
N+\tilde{N}+H+\tilde{H} & =0, \\
N e^{i k}+\tilde{N} e^{-i k}+H e^{-i k}+\tilde{H} e^{i k} & =0 .
\end{aligned}
$$

The equations are easily solved as

$$
H=-\tilde{N}, \quad N=-\tilde{H} .
$$

This gives the left reflection matrix in this section

$$
K_{l}(k)=\left(\begin{array}{cc}
0 & -1 \\
-1 & 0
\end{array}\right) .
$$


- The right boundary part,

$$
\begin{aligned}
2 \lambda^{2} n(L)-\lambda^{2} n(L-1)-\lambda^{2} \gamma & =E n(L), \\
2 \lambda^{2} h(L)-\lambda^{2} h(L-1)+\lambda^{2} \gamma & =E h(L), \\
-\lambda^{2} n(L)+\lambda^{2} h(L)+2 \lambda^{2} \gamma & =E \gamma,
\end{aligned}
$$

which imply

$$
\begin{aligned}
\gamma & =n(L+1), \\
n(L+1) & =-h(L+1), \\
h(L) & =-n(L+2) .
\end{aligned}
$$

From these equations, we can get

$$
H=-e^{-2 i k(L+1)} \tilde{N}, \quad N=-e^{-2 i k(L+1)} \tilde{H} .
$$

Then the right reflection matrix in this sector is

$$
K_{r}(k)=\left(\begin{array}{cc}
0 & -e^{-2 i k} \\
-e^{-2 i k} & 0
\end{array}\right),
$$

recalling the definition of right reflection matrix in eq. (4.46).

The compatibility of the eqs. (4.73)-(4.75) with the solutions (4.68) requires

$$
e^{i k(2 L+2)}=1, \quad k=\frac{n \pi}{L+1}, \quad n \in \mathbb{Z} .
$$

Therefore we get the Bethe equation of this sector and we also note that the effective length of the spin chain is $2 L+2$ since two more boundary excitations participate in the interaction with the bulk excitations.

The full left reflection matrix $K_{l}$ is then found to be

$$
K_{l}(k)=\left(\begin{array}{cccc}
0 & 0 & 0 & -e^{-i k} \\
0 & 0 & -1 & 0 \\
0 & -1 & 0 & 0 \\
-e^{-i k} & 0 & 0 & 0
\end{array}\right) \text {, }
$$

with the order of the excitations as $A_{2}, B_{1}^{\dagger}, A_{2}^{\dagger}, B_{1}$. And the full right reflection matrix is

$$
K_{r}(k)=\left(\begin{array}{cccc}
0 & 0 & 0 & -e^{-i k} \\
0 & 0 & -e^{-2 i k} & 0 \\
0 & -e^{-2 i k} & 0 & 0 \\
-e^{-i k} & 0 & 0 & 0
\end{array}\right) .
$$

For the spin chain associated with the operator $\hat{O}^{\prime}$, following the similar procedure shown above, we find the same reflection matrix after modifying the phase factor $e^{2 i k L}$ in the definition of right reflection matrix (4.46) into $e^{i k(2 L+1)}$. This modification is due 
to the different effective length of the open spin chain. For the same reason, the Bethe equations for the two-particle and four-particle sectors will also be slightly modified. In order to prove the integrability of the Hamiltonian, we also need to know the bulk two-loop S-matrix which has been derived in [15] using coordinate Bethe ansatz. We review this bulk S-matrix in appendix C. Equipped with the boundary and bulk scattering matrices, with the help of Mathematica program, we can verify the following reflection equations [23] ${ }^{11}$

$$
\begin{aligned}
& {\left[S\left(k_{1}, k_{2}\right)\right]_{l_{1} l_{2}}^{m_{1} m_{2}}\left[K_{l}\left(k_{2}\right)\right]_{j_{2}}^{l_{2}}\left[S\left(-k_{2}, k_{1}\right)\right]_{j_{1} i_{2}}^{l_{1} j_{2}}\left[K_{l}\left(k_{1}\right)\right]_{i_{1}}^{j_{1}}} \\
& \quad=\left[K_{l}\left(k_{1}\right)\right]_{l_{1}}^{m_{1}}\left[S\left(-k_{1}, k_{2}\right)\right]_{j_{1} l_{2}}^{l_{1} m_{2}}\left[K_{l}\left(k_{2}\right)\right]_{j_{2}}^{l_{2}}\left[S\left(-k_{2},-k_{1}\right)\right]_{i_{1} i_{2}}^{j_{1} j_{2}}, \\
& \quad=\left[S\left(-k_{1},-k_{2}\right)\right]_{l_{1} l_{2}}^{m_{1} m_{2}}\left[K_{r}\left(-k_{1}\right)\right]_{j_{1}}^{l_{1}}\left[S\left(-k_{2}, k_{1}\right)\right]_{i_{1} j_{2}}^{j_{1} l_{2}}\left[K_{r}\left(-k_{2}\right)\right]_{i_{2}}^{j_{2}} \\
& \quad=\left[K_{r}\left(-k_{2}\right)\right]_{l_{2}}^{m_{2}}\left[S\left(-k_{1}, k_{2}\right)\right]_{l_{1} j_{2}}^{m_{1} l_{2}}\left[K_{r}\left(-k_{1}\right)\right]_{j_{1}}^{l_{1}}\left[S\left(k_{2}, k_{1}\right)\right]_{i_{1} i_{2}}^{j_{1} j_{2}},
\end{aligned}
$$

which, together with the validity of (bulk) YBE, guarantee the integrability of our open spin chain.

\section{Conclusion and discussions}

In this paper, we studied the two-loop integrability of planar $\mathcal{N}=3$ flavored ABJM theory in the scalar sector. Rewriting the complete action in a manifestly $\mathrm{SU}(2)_{R}$ invariant way is the first step of the two-loop computation. Working in 't Hooft limit, we only need to compute the ADMs of composite mesonic operators which naturally correspond to states on an open alternating spin chain. Taking the 't Hooft limit also tremendously simplifies the computation of the ADMs of this class of operators since the computation for the bulk part remians the same as the one in ABJM theory. The final result of this computation can be re-expressed as a Hamiltonian on this open spin chain. The most efficient way to prove the integrability is to construct an open chain transfer matrix containing the required Hamiltonian in the framework of algebraic Bethe ansatz. A more detailed technique called projected K-matrices introduced in [25] provides a systematic way to deal with a class of systems with distinct dynamic degrees of freedom from the boundary site and the bulk like our model by the proper projection of the regular solutions of operator-valued Kmatrices. This technique has been successfully employed in [26] to obtain the left K-matrix of the projected type in [25] and eventually demonstrate the integrability of the BerensteinVazquez Hamiltonian in [14]. For our case, the first problem is to find a suitable projector for the symmetry breaking $\mathrm{SU}(4)_{R} \rightarrow \mathrm{SU}(2)_{R} \times \mathrm{SU}(2)_{D}$ and then to construct the projected $\mathrm{K}$-matrices based on the projector and the known $\mathrm{SU}(4)$ c-number K-matrices. However it is not an easy task to find the needed projected K-matrices that produce the correct boundary Hamiltonian. In the present paper, we use the more physical coordinate Bethe ansatz to show the integrability of our model. We considered one-excitation states and computed the left and right reflection matrices. Using these and the bulk two-loop Smatrix computed in [15], we verified the reflection equations for both sides of the open spin

\footnotetext{
${ }^{11}$ The reflection equations in the algebraic Bethe ansatz approach were first given in [24].
} 
chain. This established the two loop integrability of planar flavored ABJM theory in the scalar sector.

The immediate next step is to find the eigenvalues of the Hamiltonian. For this, we need to solve an eigenvalue equation constructed from the S-matrices and the reflection matrices $[22,27,28]$. To solve this eigenvalue equation, off-diagonal Bethe ansatz (ODBA) [29] seems necessary here since the reflection matrices at both sides are nondiagonal. ${ }^{12}$ One may also try the algebraic Bethe ansatz from the beginning by solving the boundary Yang-Baxter equation obtained in this approach and analyze what kind of solution could reproduce the boundary Hamiltonian. We remind that nested coordinate Bethe ansatz [27, 31] may be another choice as well.

One can also study the integrability of flavored ABJM theory in the Veneziano limit with $N_{c}, k, N_{f_{1}}, N_{f_{2}} \rightarrow \infty$ and $N_{c} / k, N_{f_{1}} / N_{c}, N_{f_{2}} / N_{c}$ fixed. In this case, the computation of the ADM will be much more complicated. For both single trace operators and mesonic operators, some Feynman diagrams previously omitted due to $N_{f_{i}} / N_{c}$ suppression should be included now. And although the mixing between certain single trace operators and flavorsinglet mesonic operators like $\sum_{s=1}^{N_{f_{2}}} Y_{s}^{\dagger} X X^{\dagger} \cdots X X^{\dagger} Y^{s}$ and $\sum_{t=1}^{N_{f_{1}}} M^{t} X^{\dagger} X \cdots X^{\dagger} X M_{t}^{\dagger}$ is $N_{f_{i}} / N_{c}$ suppressed in the 't Hooft limit, it should be taken into account in the Veneziano limit [32]. Generally speaking, we need to consider the mixing among the generalized single trace operators involving $X, X^{\dagger}, \sum_{s=1}^{N_{f_{2}}} Y^{s} Y_{s}^{\dagger}, \sum_{t=1}^{N_{f_{1}}} M_{t}^{\dagger} M^{t}$ with the color indices in the final two composite operators un-contracted.

Another interesting question is that whether the planar integrability can be generalized to the full sector of the theory and/or to higher orders of 't Hooft coupling constant $N_{c} / k$. For the four dimensional case considered in [7], as far as we know, there are no higher loop computations in the gauge theory side to obtain the higher order Hamiltonian and check the integrability at higher loop order. In the dual open string theory side, strong evidence for the integrability of the classical worldsheet theory was obtained in [33] by constructing non-local conserved charges in the full bosonic sector. This suggested that the field theory in the planar limit is integrable in the strong coupling regime. All-loop reflection matrices were determined in [34], following [35]. Symmetry can determine the reflection matrices up to at most two to-be-determined functions. For one vacuum orientation, reflection equations are automatically satisfied, while for the other vacuum orientation, reflection equations fix the ratio of the two functions to be a nature one. We take this as another strong evidence for the all-loop integrability of this theory. As for the case considered in $[8,9]$, no progress on higher loop integrability has been reported so far. Back to flavored ABJM theory, we hope the symmetry can also determine the all-order reflection matrix up to a few unknown functions. We leave all these important questions to future work.

\section{Acknowledgments}

We would like to thank Junpeng Cao, Bin Chen, Yu Jia, Yunfeng Jiang, Yupeng Wang, Fakai Wen, Jia-ju Zhang for very helpful discussions. This work was in part supported by

\footnotetext{
${ }^{12}$ Notice that ODBA has been successfully applied to the open spin chain dual to open strings between two un-parallel branes in $A d S_{5} \times S^{5}$ [30].
} 
Natural Science Foundation of China under Grant Nos. 11425522(WY), 11434013(WY), 11575202(NB, HC, JW), 11475183(NB), 11275207(HC), 11690022(HC), 11305235(SH). The work of SH is also supported by Max-Planck fellowship in Germany. The research of MZ is partly supported by INFN Iniziativa Specifica ST\&FI. NB, HC and JW thank Institute of Modern Physics, Northwest University for warm hospitality in visits during this project. JW would also like to thank the participants of the advanced workshop "Dark Energy and Fundamental Theory" supported by the Special Fund for Theoretical Physics from NSFC with Grant No. 11447613 for stimulating discussions.

\section{A Some details of $\mathcal{N}=2$ formulation}

\section{A.1 The on-shell values of auxiliary fields}

The equations of motion for the auxiliary fields in chiral multiplets give

$$
\begin{aligned}
F_{A}^{\dagger} & =\frac{4 \pi}{k}\left(W_{A} Z W-W Z W_{A}+W_{A} C E-B A W_{A}\right) \\
F^{A} & =\frac{4 \pi}{k}\left(-W^{\dagger A} Z^{\dagger} W^{\dagger}+W^{\dagger} Z^{\dagger} W^{\dagger A}-W^{\dagger A} A^{\dagger} B^{\dagger}+E^{\dagger} C^{\dagger} W^{\dagger A}\right), \\
G^{\dagger A} & =\frac{4 \pi}{k}\left(Z W Z^{A}-Z^{A} W Z+C E Z^{A}-Z^{A} B A\right) \\
G_{A} & =\frac{4 \pi}{k}\left(-Z^{\dagger} W^{\dagger} Z_{A}^{\dagger}+Z_{A}^{\dagger} W^{\dagger} Z^{\dagger}-A^{\dagger} B^{\dagger} Z_{A}^{\dagger}+Z_{A}^{\dagger} E^{\dagger} C^{\dagger}\right), \\
J_{s}^{\dagger} & =\frac{4 \pi}{k}\left(E_{s} C E+E_{s} Z W\right) \\
J^{s} & =\frac{4 \pi}{k}\left(E^{\dagger} C^{\dagger} E^{\dagger s}+W^{\dagger} Z^{\dagger} E^{\dagger s}\right) \\
H^{\dagger t} & =\frac{4 \pi}{k}\left(-A^{t} B A-A^{t} W Z\right) \\
H_{t} & =\frac{4 \pi}{k}\left(-A^{\dagger} B^{\dagger} A_{t}^{\dagger}-Z^{\dagger} W^{\dagger} A_{t}^{\dagger}\right) \\
K^{\dagger s} & =\frac{4 \pi}{k}\left(C E C^{s}+Z W C^{s}\right) \\
K_{s} & =\frac{4 \pi}{k}\left(C_{s}^{\dagger} E^{\dagger} C^{\dagger}+C_{s}^{\dagger} W^{\dagger} Z^{\dagger}\right) \\
I_{t}^{\dagger} & =\frac{4 \pi}{k}\left(-B A B_{t}-W Z B_{t}\right) \\
I^{t} & =\frac{4 \pi}{k}\left(-B^{\dagger t} A^{\dagger} B^{\dagger}-B^{\dagger \dagger} Z^{\dagger} W^{\dagger}\right)
\end{aligned}
$$

The equations of motion for the auxiliary fields in gauge multiplets give

$$
\begin{aligned}
\sigma^{n} & =\frac{2 \pi}{k} \operatorname{tr} T^{n}\left(Z Z^{\dagger}-W^{\dagger} W+C C^{\dagger}-E^{\dagger} E\right), \\
\hat{\sigma}^{n} & =\frac{2 \pi}{k} \operatorname{tr} T^{n}\left(Z^{\dagger} Z-W W^{\dagger}+A^{\dagger} A-B B^{\dagger}\right),
\end{aligned}
$$




$$
\begin{aligned}
\chi^{n} & =-\frac{4 \pi}{k} \operatorname{tr} T^{n}\left(Z \zeta^{\dagger}-\omega^{\dagger} W+C \tau^{\dagger}-v^{\dagger} E\right), \\
\bar{\chi}^{n} & =-\frac{4 \pi}{k} \operatorname{tr} T^{n}\left(\zeta Z^{\dagger}-W^{\dagger} \omega+\tau C^{\dagger}-E^{\dagger} v\right), \\
\hat{\chi}^{n} & =-\frac{4 \pi}{k} \operatorname{tr} T^{n}\left(\zeta^{\dagger} Z-W \omega^{\dagger}-B \eta^{\dagger}+\kappa^{\dagger} A\right), \\
\hat{\bar{\chi}}^{n} & =-\frac{4 \pi}{k} \operatorname{tr} T^{n}\left(Z^{\dagger} \zeta-\omega W^{\dagger}-\eta B^{\dagger}+A^{\dagger} \kappa\right),
\end{aligned}
$$

where $T^{n}, n=1, \cdots, N_{c}^{2}$, is the generator of $\mathrm{U}\left(N_{c}\right)$.

\section{A.2 The potential terms in $\mathcal{N}=2$ formulation}

The potentials from F-term and D-term contributions are given below

$$
\begin{aligned}
& -V_{D}^{\text {bos }}=-\frac{4 \pi^{2}}{k^{2}} \operatorname{tr}\left[\left(Z Z^{\dagger}+W^{\dagger} W+C C^{\dagger}+E^{\dagger} E\right)\left(Z Z^{\dagger}-W^{\dagger} W+C C^{\dagger}-E^{\dagger} E\right)\right. \\
& \left.\times\left(Z Z^{\dagger}-W^{\dagger} W+C C^{\dagger}-E^{\dagger} E\right)\right] \\
& -\frac{4 \pi^{2}}{k^{2}} \operatorname{tr}\left[\left(Z^{\dagger} Z+W W^{\dagger}+A^{\dagger} A+B B^{\dagger}\right)\left(Z^{\dagger} Z-W W^{\dagger}+A^{\dagger} A-B B^{\dagger}\right)\right. \\
& \left.\times\left(Z^{\dagger} Z-W W^{\dagger}+A^{\dagger} A-B B^{\dagger}\right)\right] \\
& +\frac{8 \pi^{2}}{k^{2}} \operatorname{tr}\left[Z_{A}^{\dagger}\left(Z Z^{\dagger}-W^{\dagger} W+C C^{\dagger}-E^{\dagger} E\right) Z^{A}\left(Z^{\dagger} Z-W W^{\dagger}+A^{\dagger} A-B B^{\dagger}\right)\right] \\
& +\frac{8 \pi^{2}}{k^{2}} \operatorname{tr}\left[W^{\dagger A}\left(Z^{\dagger} Z-W W^{\dagger}+A^{\dagger} A-B B^{\dagger}\right) W_{A}\left(Z Z^{\dagger}-W^{\dagger} W+C C^{\dagger}-E^{\dagger} E\right)\right], \\
& -V_{F}^{\text {bos }}=-\frac{16 \pi^{2}}{k^{2}} \operatorname{tr}\left(-W_{A} Z W+W Z W_{A}-W_{A} C E+B A W_{A}\right)\left(W^{\dagger A} Z^{\dagger} W^{\dagger}-W^{\dagger} Z^{\dagger} W^{\dagger A}\right. \\
& \left.+W^{\dagger A} A^{\dagger} B^{\dagger}-E^{\dagger} C^{\dagger} W^{\dagger A}\right)-\frac{16 \pi^{2}}{k^{2}} \operatorname{tr}\left(Z W Z^{A}-Z^{A} W Z+C E Z^{A}-Z^{A} B A\right) \\
& \left(-Z^{\dagger} W^{\dagger} Z_{A}^{\dagger}+Z_{A}^{\dagger} W^{\dagger} Z^{\dagger}-A^{\dagger} B^{\dagger} Z_{A}^{\dagger}+Z_{A}^{\dagger} E^{\dagger} C^{\dagger}\right)-\frac{16 \pi^{2}}{k^{2}} \operatorname{tr}\left(E_{s} C E+E_{s} Z W\right) \\
& \left(E^{\dagger} C^{\dagger} E^{\dagger s}+W^{\dagger} Z^{\dagger} E^{\dagger s}\right)-\frac{16 \pi^{2}}{k^{2}} \operatorname{tr}\left(A^{t} B A+A^{t} W Z\right)\left(A^{\dagger} B^{\dagger} A_{t}^{\dagger}+Z^{\dagger} W^{\dagger} A_{t}^{\dagger}\right) \\
& -\frac{16 \pi^{2}}{k^{2}} \operatorname{tr}\left(C E C^{s}+Z W C^{s}\right)\left(C_{s}^{\dagger} E^{\dagger} C^{\dagger}+C_{s}^{\dagger} W^{\dagger} Z^{\dagger}\right)-\frac{16 \pi^{2}}{k^{2}} \operatorname{tr}\left(B A B_{t}+W Z B_{t}\right) \\
& \left(B^{\dagger t} A^{\dagger} B^{\dagger}+B^{\dagger t} Z^{\dagger} W^{\dagger}\right), \\
& -V_{D}^{\text {ferm }}=-\frac{2 \pi i}{k} \operatorname{tr}\left(\zeta \zeta^{\dagger}+\tau \tau^{\dagger}-\omega^{\dagger} \omega-v^{\dagger} v\right)\left(Z Z^{\dagger}-W^{\dagger} W+C C^{\dagger}-E^{\dagger} E\right) \\
& +\frac{2 \pi i}{k} \operatorname{tr}\left(\zeta^{\dagger} \zeta-\omega \omega^{\dagger}-\eta \eta^{\dagger}+\kappa^{\dagger} \kappa\right)\left(Z^{\dagger} Z-W W^{\dagger}+A^{\dagger} A-B B^{\dagger}\right) \\
& -\frac{4 \pi i}{k} \operatorname{tr}\left(\zeta Z^{\dagger}-W^{\dagger} \omega+\tau C^{\dagger}-E^{\dagger} v\right)\left(Z \zeta^{\dagger}-\omega^{\dagger} W+C \tau^{\dagger}-v^{\dagger} E\right) \\
& +\frac{4 \pi i}{k} \operatorname{tr}\left(Z^{\dagger} \zeta-\omega W^{\dagger}+A^{\dagger} \kappa-\eta B^{\dagger}\right)\left(\zeta^{\dagger} Z-W \omega^{\dagger}+\kappa^{\dagger} A-B \eta^{\dagger}\right),
\end{aligned}
$$




$$
\begin{aligned}
-V_{F}^{\mathrm{ferm}}= & \operatorname{tr}\left(\frac{2 \pi}{k} \epsilon_{A C} \epsilon^{B D}\left[-2 \zeta^{A} W_{B} Z^{C} \omega_{D}-2 \zeta^{A} \omega_{B} Z^{C} W_{D}-Z^{A} \omega_{B} Z^{C} \omega_{D}-\zeta^{A} W_{B} \zeta^{C} W_{D}\right]\right. \\
& -\frac{2 \pi}{k} \epsilon^{A C} \epsilon_{B D}\left[2 \zeta_{A}^{\dagger} W^{\dagger B} Z_{C}^{\dagger} \omega^{\dagger D}+2 \zeta_{A}^{\dagger} \omega^{\dagger B} Z_{C}^{\dagger} W^{\dagger D}+Z_{A}^{\dagger} \omega^{\dagger B} Z_{C}^{\dagger} \omega^{\dagger D}+\zeta_{A}^{\dagger} W^{\dagger B} \zeta_{C}^{\dagger} W^{\dagger D}\right] \\
& -\frac{2 \pi}{k}[-2 \tau v C E-2 \tau E C v-\tau E \tau E-C v C v] \\
& +\frac{2 \pi}{k}[-2 \eta \kappa B A-2 B \kappa \eta A-B \kappa B \kappa-\eta A \eta A] \\
& +\frac{2 \pi}{k}\left[2 \kappa^{\dagger} \eta^{\dagger} A^{\dagger} B^{\dagger}+2 \kappa^{\dagger} B^{\dagger} A^{\dagger} \eta^{\dagger}+\kappa^{\dagger} B^{\dagger} \kappa^{\dagger} B^{\dagger}+A^{\dagger} \eta^{\dagger} A^{\dagger} \eta^{\dagger}\right] \\
& -\frac{2 \pi}{k}\left[2 v^{\dagger} \tau^{\dagger} E^{\dagger} C^{\dagger}+2 E^{\dagger} \tau^{\dagger} v^{\dagger} C^{\dagger}+E^{\dagger} \tau^{\dagger} E^{\dagger} \tau^{\dagger}+v^{\dagger} C^{\dagger} v^{\dagger} C^{\dagger}\right] \\
& +\frac{4 \pi}{k}\left[Z W \tau v+\zeta \omega C E+Z \omega C v+Z \omega \tau E+\zeta W C v+\zeta W^{2} E\right] \\
& +\frac{4 \pi}{k}[-W Z \eta \kappa-\omega \zeta B A-W \zeta B \kappa-W \zeta \eta A-\omega Z B \kappa-\omega Z \eta A] \\
& +\frac{4 \pi}{k}\left[A^{\dagger} B^{\dagger} \zeta^{\dagger} \omega^{\dagger}+A^{\dagger} \eta^{\dagger} Z^{\dagger} \omega^{\dagger}+A^{\dagger} \eta^{\dagger} \zeta^{\dagger} W^{\dagger}+\kappa^{\dagger} B^{\dagger} Z^{\dagger} \omega^{\dagger}+\kappa^{\dagger} B^{\dagger} \zeta^{\dagger} W^{\dagger}+\kappa^{\dagger} \eta^{\dagger} Z^{\dagger} W^{\dagger}\right] \\
& \left.+\frac{4 \pi}{k}\left[-E^{\dagger} C^{\dagger} \omega^{\dagger} \zeta^{\dagger}-E^{\dagger} \tau^{\dagger} W^{\dagger} \zeta^{\dagger}-E^{\dagger} \tau^{\dagger} \omega^{\dagger} Z^{\dagger}-v^{\dagger} C^{\dagger} W^{\dagger} \zeta^{\dagger}-v^{\dagger} C^{\dagger} \omega^{\dagger} Z^{\dagger}-v^{\dagger} \tau^{\dagger} W^{\dagger} Z^{\dagger}\right]\right)
\end{aligned}
$$

where the summation indices are suppressed for those obvious contractions between two adjacent fields.

\section{B BPS property of the reference state}

For the supersymmetry transformation of $\mathcal{N}=3$ Chern-Simon-matter theories, we follow the convention of [36]. ${ }^{13}$ We perform a Wick rotation to three dimensional Euclidean space.

The supersymmetry transformations of $Y_{i}^{\dagger}, Y^{i}, X_{i A}^{\dagger}, X^{i A}$ are

$$
\begin{aligned}
\delta Y_{i}^{\dagger} & =\mathrm{i} \psi_{j}^{\dagger} \theta_{i}^{j}, \\
\delta Y^{i} & =\mathrm{i} \theta_{j}^{i} \psi^{j}, \\
\delta X_{i A}^{\dagger} & =\mathrm{i} \xi_{j A}^{\dagger} \theta_{i}^{j}, \\
\delta X^{i A} & =\mathrm{i} \theta_{j}^{i} \xi^{j A},
\end{aligned}
$$

where the supersymmetry transformation parameters $\theta_{j}^{i}$ satisfy the constraint $\theta_{i}^{i}=0$. It is easy to see that the vacuum state

$$
|\Omega\rangle=\left|Y_{2}^{\dagger} X^{11} X_{22}^{\dagger} \cdots X^{11} X_{22}^{\dagger} Y^{1}\right\rangle
$$

is invariant under the supersymmetry transformation with $\theta_{1}^{1}=\theta_{2}^{2}=\theta_{2}^{1}=0$, so it is 1/3-BPS.

\footnotetext{
${ }^{13}$ Here we only consider the Poincare supercharges and neglect the conformal supercharges.
} 


\section{The bulk S-matrix}

In this appendix we briefly review the bulk S-matrix computed in [15]. Our convention is that in $S_{I_{1} I_{2}}^{J_{1} J_{2}}, I_{i}$ is used to denote the in-state of the $i$-th particle and $J_{i}$ is for the out-state of the $i$-th particle.

We define

$$
u_{i} \equiv \frac{1}{2} \cot \frac{k_{i}}{2}, \quad u_{i j} \equiv u_{i}-u_{j} .
$$

The non-zero elements of the bulk S-matrix is

$$
S_{\phi \phi}^{\phi \phi}\left(k_{2}, k_{1}\right)=\frac{u_{21}+i}{u_{21}-i}
$$

where $\phi$ is one of $A_{2}, B_{1}^{\dagger}, A_{2}^{\dagger}, B_{1}$;

$$
\begin{aligned}
& S_{A_{2} B_{1}^{\dagger}}^{A_{2} B^{\dagger}}\left(k_{2}, k_{1}\right)=S_{B_{1}^{\dagger} A_{2}}^{B^{\dagger} A_{2}}\left(k_{2}, k_{1}\right)=S_{A_{2}^{\dagger} B_{1}}^{A_{\dagger}^{\dagger} B_{1}}\left(k_{2}, k_{1}\right)=S_{B_{1} A_{2}^{\dagger}}^{B_{1} A_{2}^{\dagger}}\left(k_{2}, k_{1}\right)=\frac{u_{21}}{u_{21}-i} ; \\
& S_{A_{2} B_{1}^{\dagger}}^{B_{\dagger}^{\dagger} A_{2}}\left(k_{2}, k_{1}\right)=S_{B_{1}^{\dagger} A_{2}}^{A_{2} B_{1}^{\dagger}}\left(k_{2}, k_{1}\right)=S_{A_{2}^{\dagger} B_{1}}^{B_{1} A_{2}^{\dagger}}\left(k_{2}, k_{1}\right)=S_{B_{1} A_{2}^{\dagger}}^{A_{1}^{\dagger} B_{1}}\left(k_{2}, k_{1}\right)=\frac{i}{u_{21}-i} ; \\
& \left.S_{A_{2} B_{1}}^{A_{2} B_{1}}\left(k_{2}, k_{1}\right)=S_{B_{1} A_{2}}^{B_{1} A_{2}}\left(k_{2}, k_{1}\right)=S_{A_{2}^{\dagger} B_{1}^{\dagger}}^{A_{\dagger}^{\dagger} B_{2}^{\dagger}}\left(k_{2}, k_{1}\right)=S_{B_{1}^{\dagger} A_{2}^{\dagger}}^{B_{1}^{\dagger} k_{2}^{\dagger}}, k_{1}\right)=1 ; \\
& S_{A_{2} A_{2}^{\dagger}}^{A_{2} A_{2}^{\dagger}}\left(k_{2}, k_{1}\right)=S_{B_{1}^{\dagger} B_{1}}^{B_{1}^{\dagger} B_{1}}\left(k_{2}, k_{1}\right)=S_{A_{2}^{\dagger} A_{2}}^{A_{\dagger}^{\dagger} A_{2}}\left(k_{2}, k_{1}\right)=S_{B_{1} B_{1}^{\dagger}}^{B_{1} B_{1}^{\dagger}}\left(k_{2}, k_{1}\right)=\frac{u_{12}}{u_{12}-i} ; \\
& S_{B_{1}^{\dagger} B_{1}}^{A_{2} A_{2}^{\dagger}}\left(k_{2}, k_{1}\right)=S_{A_{2} A_{2}^{\dagger}}^{B_{\dagger}^{\dagger} B_{1}}\left(k_{2}, k_{1}\right)=S_{B_{1} B_{1}^{\dagger}}^{A_{\dagger}^{\dagger} A_{2}}\left(k_{2}, k_{1}\right)=S_{A_{2}^{\dagger} A_{2}}^{B_{1} B_{1}^{\dagger}}\left(k_{2}, k_{1}\right)=\frac{i}{u_{12}-i} .
\end{aligned}
$$

We also verified that this S-matrix satisfies the YBE.

Open Access. This article is distributed under the terms of the Creative Commons Attribution License (CC-BY 4.0), which permits any use, distribution and reproduction in any medium, provided the original author(s) and source are credited.

\section{References}

[1] L. Brink, J.H. Schwarz and J. Scherk, Supersymmetric Yang-Mills Theories, Nucl. Phys. B 121 (1977) 77 [INSPIRE].

[2] O. Aharony, O. Bergman, D.L. Jafferis and J.M. Maldacena, $\mathcal{N}=6$ superconformal Chern-Simons-matter theories, M2-branes and their gravity duals, JHEP 10 (2008) 091 [arXiv:0806.1218] [INSPIRE].

[3] N. Beisert et al., Review of AdS/CFT Integrability: An Overview, Lett. Math. Phys. 99 (2012) 3 [arXiv: 1012.3982] [INSPIRE].

[4] J.A. Minahan and K. Zarembo, The Bethe ansatz for $\mathcal{N}=4$ super Yang-Mills, JHEP 03 (2003) 013 [hep-th/0212208] [INSPIRE].

[5] J.A. Minahan and K. Zarembo, The Bethe ansatz for superconformal Chern-Simons, JHEP 09 (2008) 040 [arXiv:0806.3951] [INSPIRE].

[6] D. Bak and S.-J. Rey, Integrable Spin Chain in Superconformal Chern-Simons Theory, JHEP 10 (2008) 053 [arXiv: 0807.2063] [INSPIRE]. 
[7] T. Erler and N. Mann, Integrable open spin chains and the doubling trick in $\mathcal{N}=2 S Y M$ with fundamental matter, JHEP 01 (2006) 131 [hep-th/0508064] [INSPIRE].

[8] B. Chen, X.-J. Wang and Y.-S. Wu, Integrable open spin chain in super Yang-Mills and the plane wave/SYM duality, JHEP 02 (2004) 029 [hep-th/0401016] [INSPIRE].

[9] B. Chen, X.-J. Wang and Y.-S. Wu, Open spin chain and open spinning string, Phys. Lett. B 591 (2004) 170 [hep-th/0403004] [INSPIRE].

[10] S. Hohenegger and I. Kirsch, A Note on the holography of Chern-Simons matter theories with flavour, JHEP 04 (2009) 129 [arXiv:0903.1730] [INSPIRE].

[11] D. Gaiotto and D.L. Jafferis, Notes on adding D6 branes wrapping $\mathbb{R P}^{3}$ in $A d S_{4} \times \mathbb{C P}^{3}$, JHEP 11 (2012) 015 [arXiv:0903.2175] [INSPIRE].

[12] Y. Hikida, W. Li and T. Takayanagi, ABJM with Flavors and FQHE, JHEP 07 (2009) 065 [arXiv:0903.2194] [INSPIRE].

[13] D. Bak, H. Min and S.-J. Rey, Generalized Dynamical Spin Chain and 4-Loop Integrability in $\mathcal{N}=6$ Superconformal Chern-Simons Theory, Nucl. Phys. B 827 (2010) 381 [arXiv:0904.4677] [INSPIRE].

[14] D. Berenstein and S.E. Vazquez, Integrable open spin chains from giant gravitons, JHEP 06 (2005) 059 [hep-th/0501078] [INSPIRE].

[15] C. Ahn and R.I. Nepomechie, Two-loop test of the $\mathcal{N}=6$ Chern-Simons theory S-matrix, JHEP 03 (2009) 144 [arXiv:0901.3334] [INSPIRE]

[16] C. Ahn and R.I. Nepomechie, $\mathcal{N}=6$ super Chern-Simons theory S-matrix and all-loop Bethe ansatz equations, JHEP 09 (2008) 010 [arXiv:0807.1924] [INSPIRE].

[17] O. DeWolfe and N. Mann, Integrable open spin chains in defect conformal field theory, JHEP 04 (2004) 035 [hep-th/0401041] [INSPIRE].

[18] M.K. Benna, I.R. Klebanov, T. Klose and M. Smedback, Superconformal Chern-Simons Theories and $A d S_{4} / C F T_{3}$ Correspondence, JHEP 09 (2008) 072 [arXiv:0806.1519] [INSPIRE].

[19] M.K. Benna, I.R. Klebanov and T. Klose, Charges of Monopole Operators in Chern-Simons Yang-Mills Theory, JHEP 01 (2010) 110 [arXiv:0906.3008] [INSPIRE].

[20] S. He and J.-B. Wu, Note on Integrability of Marginally Deformed ABJ(M) Theories, JHEP 04 (2013) 012 [Erratum JHEP 04 (2016) 139] [arXiv:1302.2208] [INSPIRE].

[21] H.-H. Chen, P. Liu and J.-B. Wu, Y-system for $\gamma$-deformed ABJM theory, JHEP 03 (2017) 133 [arXiv: 1611.02804] [INSPIRE].

[22] Y.-Y. Li, J. Cao, W.-L. Yang, K. Shi and Y. Wang, Exact solution of the one-dimensional Hubbard model with arbitrary boundary magnetic fields, Nucl. Phys. B 879 (2014) 98 [arXiv:1311.0432] [INSPIRE].

[23] I.V. Cherednik, Factorizing Particles on a Half Line and Root Systems, Theor. Math. Phys. 61 (1984) 977 [Teor. Mat. Fiz. 61 (1984) 35] [INSPIRE].

[24] E.K. Sklyanin, Boundary Conditions for Integrable Quantum Systems, J. Phys. A 21 (1988) 2375 [INSPIRE].

[25] H. Frahm and N.A. Slavnov, New solutions to the reflection equation and the projecting method, J. Phys. A 32 (1999) 1547 [cond-mat/9810312]. 
[26] R.I. Nepomechie, Revisiting the $Y=0$ open spin chain at one loop, JHEP 11 (2011) 069 [arXiv:1109.4366] [INSPIRE].

[27] H. Schulz, Hubbard chain with reflecting ends, J. Phys. C 18 (1985) 581.

[28] N. Andrei, K. Furuya and J.H. Lowenstein, Solution of the Kondo Problem, Rev. Mod. Phys. 55 (1983) 331 [INSPIRE].

[29] Y. Wang, W.-L. Yang, J. Cao and K. Shi, Off-Diagonal Bethe Ansatz for Exactly Solvable Models, Springer Press (2015).

[30] X. Zhang et al., Bethe ansatz for an AdS/CFT open spin chain with non-diagonal boundaries, JHEP 10 (2015) 133 [arXiv:1507.08866] [INSPIRE].

[31] D.H. Correa and C.A.S. Young, Asymptotic Bethe equations for open boundaries in planar AdS/CFT, J. Phys. A 43 (2010) 145401 [arXiv:0912.0627] [InSPIRE].

[32] A. Gadde, E. Pomoni and L. Rastelli, Spin Chains in $\mathcal{N}=2$ Superconformal Theories: From the $\mathbb{Z}_{2}$ Quiver to Superconformal QCD, JHEP 06 (2012) 107 [arXiv: 1006.0015] [INSPIRE].

[33] N. Mann and S.E. Vazquez, Classical Open String Integrability, JHEP 04 (2007) 065 [hep-th/0612038] [INSPIRE].

[34] D.H. Correa and C.A.S. Young, Reflecting magnons from D7 and D5 branes, J. Phys. A 41 (2008) 455401 [arXiv: 0808.0452] [INSPIRE].

[35] D.M. Hofman and J.M. Maldacena, Reflecting magnons, JHEP 11 (2007) 063 [arXiv:0708.2272] [INSPIRE].

[36] H. Ouyang, J.-B. Wu and J.-j. Zhang, Construction and classification of novel BPS Wilson loops in quiver Chern-Simons-matter theories, Nucl. Phys. B 910 (2016) 496 [arXiv: 1511.02967] [INSPIRE]. 